PITHA 00/20

hep-ph/0008255

24 August 2000

\title{
Symmetry-breaking corrections to heavy-to-light $B$ meson form factors at large recoil
}

\author{
M. Beneke and Th. Feldmann \\ Institut für Theoretische Physik E, RWTH Aachen, 52056 Aachen, Germany
}

\begin{abstract}
Recently it has been shown that symmetries emerging in the heavy quark and large recoil energy limit impose various relations on form factors that parametrise the decay of $B$ mesons into light mesons. These symmetries are broken by perturbative effects. In this paper we discuss the structure of heavy-to-light form factors including such effects and compute symmetry-breaking corrections to first order in the strong coupling. As an application of our results we consider the forwardbackward asymmetry zero in the rare decay $B \rightarrow V \ell^{+} \ell^{-}$and the possibility to constrain potential new physics contributions to the Wilson coefficient $C_{9}$.
\end{abstract}

(submitted to Nucl. Phys. B) 


\section{Introduction}

The form factors which we discuss in this paper are matrix elements of bilinear quark currents between a $B$ meson and a light pseudoscalar or vector meson. These form factors encode strong interaction effects in exclusive, semi-leptonic or radiative $B$ decays, such as $B \rightarrow \pi \ell \nu, B \rightarrow K^{*} \gamma$ etc. They also appear as non-perturbative parameters in the factorization theorem for non-leptonic $B$ decays in the heavy quark mass limit [1, 2]. The knowledge of these form factors therefore helps us to determine the CKM coupling $\left|V_{u b}\right|$, and to predict $\mathrm{CP}$ violating asymmetries and other quantities in rare $B$ decays.

Form factors for heavy-light transitions are presumably dominated by QCD interactions at small momentum transfer and therefore not computable in perturbation theory. Charles et al. have shown that certain symmetries apply to this soft contribution, when the momentum of the final light meson is large [3]. These symmetries reduce the number of independent form factors from ten to three, but they are broken by radiative corrections. In this paper we give a brief derivation of these large recoil symmetry relations and then compute the symmetry-breaking corrections at first order in the strong coupling constant $\alpha_{s}$. (At small recoil the standard heavy quark symmetries apply [4, 5]. We do not discuss this kinematic region in this paper.) This can be done since symmetrybreaking corrections arise only from short distances. An interesting application of our result is the forward-backward asymmetry in the rare decay $B \rightarrow V \ell^{+} \ell^{-}$(where $V$ is a vector meson and $\ell$ a lepton). We show that a measurement of the lepton-invariant mass squared, where this asymmetry vanishes, yields a direct measurement of the loop induced Wilson coefficient $C_{9}$ in the weak effective Hamiltonian [6], which is almost free of hadronic uncertainties even after including $\alpha_{s}$-corrections. This generalises an observation recently made by Ali et al. [7].

\section{Derivation and discussion of large-recoil symme- tries}

The form factors for $\bar{B}$ decays into a pseudoscalar meson are defined by the following Lorentz decompositions of bilinear quark current matrix elements:

$$
\begin{gathered}
\left\langle P\left(p^{\prime}\right)\left|\bar{q} \gamma^{\mu} b\right| \bar{B}(p)\right\rangle=f_{+}\left(q^{2}\right)\left[p^{\mu}+p^{\prime \mu}-\frac{M^{2}-m_{P}^{2}}{q^{2}} q^{\mu}\right]+f_{0}\left(q^{2}\right) \frac{M^{2}-m_{P}^{2}}{q^{2}} q^{\mu}, \\
\left\langle P\left(p^{\prime}\right)\left|\bar{q} \sigma^{\mu \nu} q_{\nu} b\right| \bar{B}(p)\right\rangle=\frac{i f_{T}\left(q^{2}\right)}{M+m_{P}}\left[q^{2}\left(p^{\mu}+p^{\prime \mu}\right)-\left(M^{2}-m_{P}^{2}\right) q^{\mu}\right],
\end{gathered}
$$

where $M$ is the $B$ meson mass, $m_{P}$ the mass of the pseudoscalar meson and $q=p-p^{\prime}$. The relevant form factors for $B$ decays into vector mesons are defined as

$$
\begin{gathered}
\left\langle V\left(p^{\prime}, \varepsilon^{*}\right)\left|\bar{q} \gamma^{\mu} b\right| \bar{B}(p)\right\rangle=\frac{2 i V\left(q^{2}\right)}{M+m_{V}} \epsilon^{\mu \nu \rho \sigma} \varepsilon_{\nu}^{*} p_{\rho}^{\prime} p_{\sigma} \\
\left\langle V\left(p^{\prime}, \varepsilon^{*}\right)\left|\bar{q} \gamma^{\mu} \gamma_{5} b\right| \bar{B}(p)\right\rangle=2 m_{V} A_{0}\left(q^{2}\right) \frac{\varepsilon^{*} \cdot q}{q^{2}} q^{\mu}+\left(M+m_{V}\right) A_{1}\left(q^{2}\right)\left[\varepsilon^{* \mu}-\frac{\varepsilon^{*} \cdot q}{q^{2}} q^{\mu}\right]
\end{gathered}
$$




$$
\begin{gathered}
-A_{2}\left(q^{2}\right) \frac{\varepsilon^{*} \cdot q}{M+m_{V}}\left[p^{\mu}+p^{\prime \mu}-\frac{M^{2}-m_{V}^{2}}{q^{2}} q^{\mu}\right], \\
\left\langle V\left(p^{\prime}, \varepsilon^{*}\right)\left|\bar{q} \sigma^{\mu \nu} q_{\nu} b\right| \bar{B}(p)\right\rangle=2 T_{1}\left(q^{2}\right) \epsilon^{\mu \nu \rho \sigma} \varepsilon_{\nu}^{*} p_{\rho} p_{\sigma}^{\prime}, \\
\left\langle V\left(p^{\prime}, \varepsilon^{*}\right)\left|\bar{q} \sigma^{\mu \nu} \gamma_{5} q_{\nu} b\right| \bar{B}(p)\right\rangle=(-i) T_{2}\left(q^{2}\right)\left[\left(M^{2}-m_{V}^{2}\right) \varepsilon^{* \mu}-\left(\varepsilon^{*} \cdot q\right)\left(p^{\mu}+p^{\prime \mu}\right)\right] \\
+(-i) T_{3}\left(q^{2}\right)\left(\varepsilon^{*} \cdot q\right)\left[q^{\mu}-\frac{q^{2}}{M^{2}-m_{V}^{2}}\left(p^{\mu}+p^{\prime \mu}\right)\right],
\end{gathered}
$$

where $m_{V}(\varepsilon)$ is the mass (polarisation vector) of the vector meson and we use the sign convention $\epsilon^{0123}=-1$.

We begin with a qualitative discussion of strong interaction symmetries and radiative corrections for form factors in $B$ meson decay in the limit $M / \Lambda_{\mathrm{QCD}} \rightarrow \infty$. $\left(\Lambda_{\mathrm{QCD}}\right.$ is the strong interaction scale.) It is useful to briefly recapitulate the implications of heavy quark symmetry, when the final meson $P(V)$ is also heavy, for example a $D$ meson.

\subsection{Recapitulation: heavy-heavy form factors}

As long as the velocity transfer to the $D$ meson remains of order 1 , we may assume that the heavy quarks interact with the spectator quark (and other soft degrees of freedom) exclusively via soft exchanges characterised by momentum transfers much smaller than the heavy quark masses. Any hard interaction would imply large momentum of the spectator quark in the $B$ meson or $D$ meson or both, and such a configuration is assumed to be highly improbable. The simplifications that occur when heavy quarks interact only with soft gluons are formalised as heavy quark effective theory (HQET) [8, 9, 10, 11]. The heavy quark momentum $p_{Q}(Q=b, c)$ is decomposed into a large "kinematic" term and a small residual momentum $\left(k^{\mu}\right)$,

$$
p_{Q}^{\mu}=m_{Q} v^{\mu}+k^{\mu}, \quad|k| \ll m_{Q},
$$

where $v$ is the heavy meson velocity. To leading order in $\Lambda_{\mathrm{QCD}} / m_{Q}$, the interaction of heavy quarks with soft gluons is described by the effective lagrangian

$$
\mathcal{L}_{\mathrm{HQET}}=\bar{Q}_{v}(i v \cdot D) Q_{v}+O\left(1 / m_{Q}\right)
$$

Here $Q_{v}(x)=e^{i m_{Q} v \cdot x} \frac{1+\phi}{2} Q(x)$ denotes the large components of the heavy quark spinor field with its leading $m_{Q^{-}}$-dependence made explicit, and $D^{\mu}=\partial^{\mu}-i g_{s} A^{\mu}$ is the covariant derivative in QCD.

Eq. (8) implies the well-known spin and heavy flavour symmetries which arise in the infinite quark mass limit [4, 5]. A consequence of these symmetries is that the three pseudoscalar and seven vector form factors defined in Eqs. (11)-(6) are all related to a single function of velocity transfer $v \cdot v^{\prime}, \xi\left(v \cdot v^{\prime}\right)$, whose absolute normalization is known at zero recoil $(\xi(1)=1)$ owing to current conservation. The heavy quark symmetries are violated by radiative corrections (as well as higher dimension operators in the effective 


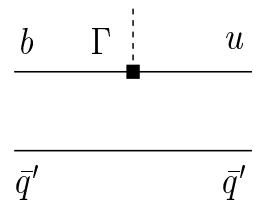

(a)

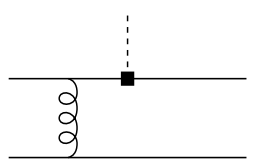

(c)

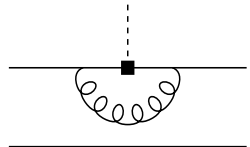

(b)

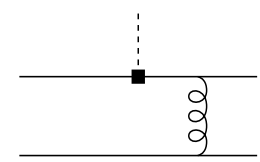

(d)

Figure 1: Different contributions to the $B \rightarrow P(V)$ transition. (a) Soft contribution (soft interactions with the spectator antiquark $\bar{q}^{\prime}$ are not drawn). (b) Hard vertex renormalisation. (c,d) Hard spectator interaction.

lagrangian), such as the one shown in Fig. 1 $1 \mathrm{~b}$. (The disconnected spectator quark line in Figs. 1 $1 \mathrm{a}$ and $1 \mathrm{~b}$ is meant to indicate that it is connected to the other lines only via soft exchanges. Fig. 17a therefore stands for the leading term in the heavy quark mass limit.) The symmetry-breaking effects are caused only by the short-distance part of Fig. 1 b. They are accounted for by multiplicatively renormalising the heavy quark current in HQET, $[\bar{c} \Gamma b]_{\mathrm{QCD}}=\sum_{\Gamma^{\prime}} C_{\Gamma \Gamma^{\prime}}\left(v \cdot v^{\prime}, \alpha_{s}\right)\left[c_{v^{\prime}} \Gamma^{\prime} b_{v}\right]_{\mathrm{HQET}}$. Hence, neglecting $1 / m_{Q}$ corrections, there remain nine parameter-free relations between the pseudoscalar and vector form factors.

The assumption that any interaction with the spectator quark is soft means that diagrams such as those in Fig. 1 $\mathrm{c}$ and 1 d must vanish, when some of the gluon's momentum components stay finite in the heavy quark mass limit. This is clearly an approximation, whose validity depends on the behaviour of the $B$ and $D$ meson wave functions. A nonvanishing contribution from these diagrams cannot be accounted for by multiplicative renormalisation of the heavy quark currents. In fact, it is not accounted for to any order in the heavy quark expansion in the HQET formalism. As we shall see, a non-vanishing contribution from "hard spectator interactions" is one of the main differences between heavy-heavy form factors and heavy-light form factors at large recoil.

\subsection{Heavy-light form factors at large recoil}

We now turn to decays into light mesons and require the light meson to have energy $E=\left(M^{2}+m^{2}-q^{2}\right) /(2 M)$ of order $M / 2$. More precisely, we require that $E-M / 2 \ll M$ or, equivalently, $q^{2} \ll M^{2}$. Let us continue to assume that the $b$ quark and the energetic $u$ quark, created in the $b \rightarrow u$ transition, interact with the spectator quark (and other soft degrees of freedom) exclusively via soft exchanges. We may then continue to use Eq. (8) for the $b$ quark. A similar simplification occurs for the energetic light quark, for which we may use the eikonal approximation.

We introduce a light-like vector $n_{-}^{\mu}\left(n_{-}^{2}=0\right)$ parallel to the four-momentum $p^{\prime}$ of 
the light meson. (In the following we always neglect effects quadratic in the light meson mass, so that $p^{\prime 2} \approx 0$. We retain "kinematic" corrections linear in $m_{P}$ or $m_{V}$ introduced through the definition of the form factors.) Since we are discussing the soft contribution to the form factor, the $u$ quark created in the decay of the $b$ quark carries almost all the energy of the light meson, while the spectator quark is soft. Hence, we write

$$
p_{u}^{\prime \mu}=E n_{-}^{\mu}+k^{\prime \mu}, \quad\left|k^{\prime}\right| \ll E,
$$

where $p_{u}^{\prime}$ is the momentum of the $u$ quark and $k^{\prime \mu}$ is a small residual momentum. To leading order in $\Lambda_{\mathrm{QCD}} / E$, the interaction of energetic quarks with soft gluons is described by the eikonal lagrangian [12, 3]

$$
\mathcal{L}_{\text {eik }}=\bar{q}_{n} \frac{\not \phi_{+}}{2}\left(i n_{-} \cdot D\right) q_{n}+O\left(1 / E_{q}\right)
$$

where $q_{n}(x)=e^{i E_{q} n_{-} \cdot x} \frac{\underline{h_{-}} \underline{h}_{+}}{4} q(x)$ are the large components of the light quark spinor field. Here $n_{+}=2 v-n_{-}$is another light-like vector with $n_{+} \cdot n_{-}=2$ and $E_{q} \approx E$ is the energy of the light quark. (The factor $\not_{+} / 2$ in Eq. (10) can be omitted in the calculation of on-shell correlation functions.)

In the large recoil limit, the combination of Eqs. (8) and (10) implies non-trivial relations between the soft contributions to the form factors [3], which we rederive below. There is an important distinction between the effective lagrangian for heavy quarks and for energetic light quarks. The effective lagrangian (10) applies to light mesons produced in an asymmetric configuration, in which a single quark carries almost all momentum. Even for light-cone dominated processes this is an atypical configuration (the preferred one having nearly equal momentum of the quark and antiquark), not speaking of the wave functions that diagonalise the strong interaction hamiltonian. For this reason, although the interaction (10) is spin-symmetric, the symmetry is not realised in the hadronic spectrum, and there exists no relation between the soft contributions to the form factors of pseudoscalar and vector mesons. Furthermore, the probability that such an asymmetric parton configuration hadronises into a light meson depends on the energy of the meson. Hence, the soft contributions to the form factors are energy-dependent functions, whose absolute normalization is not known. This is to be contrasted to the case of heavy-heavy form factors, for which spin symmetry relates pseudoscalar and vector mesons, and for which the Isgur-Wise form factor $\xi\left(v \cdot v^{\prime}\right)$ is independent on the heavy quark mass.

To work out the large-recoil symmetry constraints on the soft form factor, we use a technique familiar from HQET. Continuing to neglect hard interactions, we write $[\bar{q} \Gamma b]_{\mathrm{QCD}}=\left[\bar{q}_{n} \Gamma b_{v}\right]_{\mathrm{eff}}$. The form factors at large recoil are then represented by

$$
\left\langle L\left(E n_{-}\right)\left|\bar{q}_{n} \Gamma b_{v}\right| B(M v)\right\rangle=\operatorname{tr}\left[A_{L}(E) \overline{\mathcal{M}}_{\mathrm{L}} \Gamma \mathcal{M}_{\mathrm{B}}\right]
$$

where $L=P, V$ and

$$
\overline{\mathcal{M}}_{\mathrm{L}}=\left\{\begin{array}{c}
\left(-\gamma_{5}\right) \\
\notin^{*}
\end{array}\right\} \frac{\not \phi_{+} \not \phi_{-}}{4} \quad \begin{aligned}
& L=P \\
& L=V
\end{aligned} \quad \mathcal{M}_{\mathrm{B}}=\frac{1+\not}{2}\left(-\gamma_{5}\right)
$$


with $\varepsilon$ the polarisation vector of the vector meson. (The generalisation of $\mathcal{M}_{\mathrm{B}}$ to the case of a vector $B$ meson is obvious. However, in this paper we restrict ourselves to pseudoscalar $B$ mesons. In Eq. (11) we assume the conventional relativistic normalisation of the states. The factor $\sqrt{M}$ which would then normally appear in $\mathcal{M}_{\mathrm{B}}$ is absorbed into $A_{L}(E)$.) The function $A_{L}(E)$ contains the long-distance dynamics, but it is independent on the Dirac structure $\Gamma$ of the current, because the effective lagrangians (8) and (10) do not contain a Dirac matrix. The most general form $A_{L}(E)$ can take is therefore

$$
A_{L}(E)=a_{1 L}(E)+a_{2 L}(E) \not b+a_{3 L}(E) \not \phi_{-}+a_{4 L}(E) \not \phi_{-} \not
$$

but the projectors $\overline{\mathcal{M}}_{\mathrm{L}}, \mathcal{M}_{\mathrm{B}}$ imply that not all the $a_{i L}(E)$ are independent. Accounting for these projectors, the most general form is

$$
\begin{aligned}
& A_{P}(E)=2 E \xi_{P}(E), \\
& A_{V}(E)=E \not \not_{-}\left(\xi_{\perp}(E)-\frac{\psi}{2} \xi_{\|}(E)\right)
\end{aligned}
$$

with a conveniently chosen overall normalisation. It follows that the three pseudoscalar meson form factors are all related to a single function $\xi_{P}(E)$ and the seven vector meson form factors are all related to two unknown functions, $\xi_{\perp}(E)$ and $\xi_{\|}(E)$. The latter two functions are chosen such that only $\xi_{\perp}(E)$ contributes the form factors for a transversely polarised vector meson and only $\xi_{\|}(E)$ contributes the production of a longitudinally polarised vector meson. Performing the trace in Eq. (11), we obtain

$$
\begin{aligned}
& \left\langle P\left(p^{\prime}\right)\left|\bar{q} \gamma^{\mu} b\right| \bar{B}(p)\right\rangle=2 E \xi_{P}(E) n_{-}^{\mu}, \\
& \left\langle P\left(p^{\prime}\right)\left|\bar{q} \sigma^{\mu \nu} q_{\nu} b\right| \bar{B}(p)\right\rangle=2 i E \xi_{P}(E)\left((M-E) n_{-}^{\mu}-M v^{\mu}\right)
\end{aligned}
$$

for pseudoscalar mesons, and

$$
\begin{aligned}
& \left\langle V\left(p^{\prime}, \varepsilon^{*}\right)\left|\bar{q} \gamma^{\mu} b\right| \bar{B}(p)\right\rangle=2 i E \xi_{\perp}(E) \epsilon^{\mu \nu \rho \sigma} \varepsilon_{\nu}^{*} n_{-\rho} v_{\sigma} \\
& \left\langle V\left(p^{\prime}, \varepsilon^{*}\right)\left|\bar{q} \gamma^{\mu} \gamma_{5} b\right| \bar{B}(p)\right\rangle=2 E\left(\xi_{\perp}(E)\left(\varepsilon^{* \mu}-\varepsilon^{*} \cdot v n_{-}^{\mu}\right)+\xi_{\|}(E) \varepsilon^{*} \cdot v n_{-}^{\mu}\right), \\
& \left\langle V\left(p^{\prime}, \varepsilon^{*}\right)\left|\bar{q} \sigma^{\mu \nu} q_{\nu} b\right| \bar{B}(p)\right\rangle=2 E M \xi_{\perp}(E) \epsilon^{\mu \nu \rho \sigma} \varepsilon_{\nu}^{*} v_{\rho} n_{-\sigma}, \\
& \left\langle V\left(p^{\prime}, \varepsilon^{*}\right)\left|\bar{q} \sigma^{\mu \nu} \gamma_{5} q_{\nu} b\right| \bar{B}(p)\right\rangle=(-2 i E)\left[\xi_{\perp}(E) M\left(\varepsilon^{* \mu}-\varepsilon^{*} \cdot v n_{-}^{\mu}\right)\right. \\
& \left.+\xi_{\|}(E) \varepsilon^{*} \cdot v\left((M-E) n_{-}^{\mu}-M v^{\mu}\right)\right]
\end{aligned}
$$

for vector mesons, in agreement with Ref. [3]. (Note, however, the different convention for the longitudinal form factor with $\xi_{\|}(E)=m_{V} / M \zeta_{\|}(E), \zeta_{\|}(E)$ being defined in Ref. [3]. We also neglect some $m_{V}^{2} / M^{2}$ terms that should not be kept at leading order in $1 / M$.) Comparing Eqs. (11)-(6) with Eqs. (16)-(21), we find the following form factor relations:

$$
f_{+}\left(q^{2}\right)=\frac{M}{2 E} f_{0}\left(q^{2}\right)=\frac{M}{M+m_{P}} f_{T}\left(q^{2}\right)=\xi_{P}(E)
$$


for pseudoscalar mesons and

$$
\begin{gathered}
\frac{M}{M+m_{V}} V\left(q^{2}\right)=\frac{M+m_{V}}{2 E} A_{1}\left(q^{2}\right)=T_{1}\left(q^{2}\right)=\frac{M}{2 E} T_{2}\left(q^{2}\right)=\xi_{\perp}(E), \\
\frac{m_{V}}{E} A_{0}\left(q^{2}\right)=\frac{M+m_{V}}{2 E} A_{1}\left(q^{2}\right)-\frac{M-m_{V}}{M} A_{2}\left(q^{2}\right)=\frac{M}{2 E} T_{2}\left(q^{2}\right)-T_{3}\left(q^{2}\right)=\xi_{\|}(E)
\end{gathered}
$$

for vector mesons. These relations are valid for the soft contribution to the form factors at large recoil, neglecting corrections of order $1 / m_{b}$ and $\alpha_{s}$. (The $\alpha_{s}$ corrections will be computed below.)

We must now examine more carefully our assumption that the $b$ quark and the $u$ quark created at the weak interaction vertex interact with the spectator quark exclusively via the exchange of soft gluons. As in the case of a heavy-to-heavy transition, there will be a vertex correction (of the type shown at one loop in Fig. 四b). The hard part of this vertex correction does not respect the symmetry relations, but it can be accounted for in perturbation theory by multiplicatively renormalising the current $\left[u_{n} \Gamma b_{v}\right]_{\mathrm{eff}}$ in the effective theory just as in the case of a heavy-to-heavy transition. There is an additional complication compared to the heavy-heavy case, because there is a long-distance sensitive contribution from energetic gluons whose momentum is collinear to the momentum of the outgoing $u$ quark. This "hard-collinear" contribution is not naturally part of the soft form factor, neither is it perturbatively computable. We shall discuss this in some more detail later, but the structure of the argument does not change due to this extra contribution.

The important new element of the discussion is provided by the hard spectator interaction shown in Figs. 1c and 1 d d. In the absence of a hard spectator interaction, the light meson is produced in a parton configuration, in which the $u$ quark carries all momentum of the meson, up to an amount of order $\Lambda_{\mathrm{QCD}}$ in the $B$ meson rest frame. In contrast, a hard interaction with the spectator quark allows the meson to be formed in a preferred configuration, in which the momentum is distributed nearly equally between the two quarks. To estimate the relative importance of the two contributions, we need to know the amplitude for producing a light meson in an asymmetric configuration.

We consider first the hard contribution. (A more extensive version of the following discussion can be found in Sect. 3.2 of [2].) Since both quarks that form the light meson have momentum of order $M$ by assumption, and the gluon in Figs. 11c and 1 $\mathrm{d}$ has virtuality of order $M \Lambda_{\mathrm{QCD}}$, this contribution can be computed by means of the hardscattering approach to exclusive processes [13, 14]. We shall do this explicitly below; the resulting scaling behaviour for the pseudoscalar meson form factors is

$$
f_{+, 0, T ; \text { hard }}\left(q^{2} \approx 0\right) \sim \alpha_{s}\left(\sqrt{M \Lambda_{\mathrm{QCD}}}\right)\left(\frac{\Lambda_{\mathrm{QCD}}}{M}\right)^{3 / 2}
$$

The scaling law for the soft contribution can be derived in different ways, but all of them make use of the endpoint behaviour of the pion's light-cone distribution amplitude [13. If we assume that the distribution amplitude vanishes linearly when the longitudinal momentum fraction of the spectator quark approaches zero, as is suggested by its 
asymptotic form, we find

$$
f_{+, 0, T ; \operatorname{soft}}\left(q^{2} \approx 0\right) \sim \xi_{P}(E \approx M / 2) \sim \sqrt{\frac{M}{E}}\left(\frac{\Lambda_{\mathrm{QCD}}}{E}\right)^{3 / 2} \sim\left(\frac{\Lambda_{\mathrm{QCD}}}{M}\right)^{3 / 2} .
$$

(This scaling law was first derived in the context of QCD sum rules [15]. Recent evaluations of the sum rule including radiative corrections [16, 17] also agree with Eqs. (25), (26).) The hard and soft contributions to Figs. 1 $\mathrm{c}$ and 1 d are not separately well-defined. The hard-scattering kernel has a logarithmic endpoint divergence [18, 19]; one must introduce a factorisation scale and factorise the endpoint divergence into the soft form factor $\xi_{P}(E)$. (In the context of QCD sum rules this point is also discussed in [17].). We can summarize this discussion by the following, tentative, factorization formula for a heavy-light form factor at large recoil, and at leading order in $1 / M$ :

$$
f_{i}\left(q^{2}\right)=C_{i} \xi_{P}(E)+\Phi_{B} \otimes T_{i} \otimes \Phi_{P}
$$

where $\xi_{P}(E)$ is the soft part of the form factor, to which the symmetries discussed above apply; $T_{i}$ is a hard-scattering kernel (with the endpoint divergence regulated in a certain manner), convoluted with the light-cone distribution amplitudes of the $B$ meson and the light pseudoscalar meson; $C_{i}=1+O\left(\alpha_{s}\right)$ is the hard vertex renormalisation (including, at present, the hard-collinear contribution discussed above). Eq. (25) implies that the hard spectator interaction (Figs. 1]c and 11d) is suppressed by one power of $\alpha_{s}$ relative to the soft contribution (Fig. [1a). Hence the form factor relation (22) is indeed correct at leading order in $1 / M$ and $\alpha_{s}$. The correction at order $\alpha_{s}$ can be computed in terms of a hard vertex renormalisation and the hard spectator interaction diagrams. This will be done explicitly in Sect. 3 .

The previous discussion applies unmodified to form factors of transversely polarised vector mesons. For longitudinally polarised vector mesons, we need to keep in mind that $\xi_{\|}(E) / \xi_{\perp}(E) \sim m_{V} / E$. Therefore $m_{V} / E \times A_{0}, A_{2}$ and $T_{3}$ scale as $\left(\Lambda_{\mathrm{QCD}} / M\right)^{5 / 2}$, when one considers longitudinally polarised vector mesons. But since the longitudinal polarisation vector is enhanced by a factor $E / m_{V}$ in the large-energy limit, this implies that the form factors above times the polarisation vectors and the form factors of transversely polarised vector mesons all follow the same scaling laws in the heavy quark limit, and they all obey a factorisation formula analogous to Eq. (27). In particular, the two terms of Eqs. (19) and (21) containing $\xi_{\|}(E)$ and $\xi_{\perp}(E)$, respectively, both scale as $M^{1 / 2} \Lambda_{\mathrm{QCD}}^{3 / 2}$ in the heavy quark/large energy limit.

Our discussion up to now has ignored the possibility that the configuration in which one quark carries almost all momentum, i.e. the soft contribution to the form factors, may be suppressed by a Sudakov form factor. If this were the case, then the term $C_{i} \xi_{P}(E)$ in Eq. (27) would be subleading compared to the hard spectator term $\Phi_{B} \otimes T_{i} \otimes \Phi_{P}$, and perhaps it could be ignored entirely. In this situation the form factor would be computable completely in the hard-scattering approach and the symmetry relations (22)(24) would seem to be irrelevant. This is assumed, for example, by the treatment of the $B \rightarrow \pi$ form factors in Refs. [20, 21]. Since in reality the energy of the outgoing light quark is not particularly large (around $2.5 \mathrm{GeV}$ ), it is equally possible that the Sudakov form factor does not suppress the soft contribution sufficiently, so that the symmetry 
relations remain approximately valid. As our default power counting we shall therefore take the case that the soft contribution dominates by one power of $\alpha_{s}$, as discussed above.

\section{Calculation of symmetry-breaking corrections}

In this section we compute the corrections of order $\alpha_{s}$ to the symmetry relations (16)(24). As discussed above these are of two distinct origins and we begin with the more familiar case of vertex renormalisation (Fig. 1 b), and then turn to the hard spectator interaction.

\subsection{Vertex corrections}

The one-loop diagram in Fig. 1 b contains ultraviolet and infrared divergences. The UV divergences are treated by dimensional regularisation $(d=4-2 \epsilon)$; the IR divergences are regulated by introducing a (small) mass term $\lambda$ for the gluon, and then factored into the soft form factor.

Using standard techniques, we obtain for a generic Dirac structure $\Gamma$ at the heavyto-light vertex the following result for the one-loop vertex correction:

$$
\begin{aligned}
& \bar{u}\left(p^{\prime}\right) \Gamma\left(p^{\prime}, p\right) u(p)=\frac{\alpha_{s} C_{F}}{4 \pi} \bar{u}\left(p^{\prime}\right)\left[\left\{-\frac{1}{2} \ln ^{2} \frac{\lambda^{2} m_{b}^{2}}{\left(m_{b}^{2}-q^{2}\right)^{2}}-2 \ln \frac{\lambda^{2} m_{b}^{2}}{\left(m_{b}^{2}-q^{2}\right)^{2}}\right.\right. \\
& \left.-2 \operatorname{Li}_{2}\left[\frac{q^{2}}{m_{b}^{2}}\right]-2 \frac{m_{b}^{2}}{q^{2}} \ln \left[1-\frac{q^{2}}{m_{b}^{2}}\right]-3-\frac{\pi^{2}}{2}\right\} \Gamma \\
& +\frac{1}{4}\left\{\frac{1}{\hat{\epsilon}}+3-\ln \frac{m_{b}^{2}}{\mu^{2}}-\left(1-\frac{m_{b}^{2}}{q^{2}}\right) \ln \left[1-\frac{q^{2}}{m_{b}^{2}}\right]\right\} \gamma^{\alpha} \gamma^{\beta} \Gamma \gamma_{\beta} \gamma_{\alpha} \\
& +\frac{q^{2}+m_{b}^{2} \ln \left[1-\frac{q^{2}}{m_{b}^{2}}\right]}{2 q^{4}} \gamma^{\alpha} \not p \Gamma \not p^{\prime} \gamma_{\alpha}+\frac{q^{2}+\left(m_{b}^{2}-q^{2}\right) \ln \left[1-\frac{q^{2}}{m_{b}^{2}}\right]}{2 q^{4}} m_{b} \gamma^{\alpha} \not p \Gamma \gamma_{\alpha} \\
& \left.-\frac{q^{2}+\left(m_{b}^{2}-2 q^{2}\right) \ln \left[1-\frac{q^{2}}{m_{b}^{2}}\right]}{q^{4}} m_{b} \Gamma \not p^{\prime}\right] u(p),
\end{aligned}
$$

where $\bar{u}\left(p^{\prime}\right)$ and $u(p)$ denote the external Dirac spinors for the light and heavy quark, respectively, and we have defined $1 / \hat{\epsilon} \equiv 1 / \epsilon-\gamma_{E}+\ln 4 \pi$. For a given current $\Gamma$, the product $\gamma^{\alpha} \gamma^{\beta} \Gamma \gamma_{\beta} \gamma_{\alpha}$ is evaluated in the naive dimensional regularisation (NDR) scheme with anticommuting $\gamma_{5}$, and the $1 / \hat{\epsilon}$ pole is then subtracted. This corresponds to defining the bilinear quark current matrix elements in the $\overline{\mathrm{MS}} / \mathrm{NDR}$ scheme.

The coefficients $C_{i}$ in Eq. (27) would normally be obtained by computing the oneloop vertex correction in the HQET/eikonal effective theory, using the same infrared regularisation as in the full theory calculation above. The one-loop correction to $C_{i}$ is

simply the difference between the two calculations and if both theories have the same 
infrared behaviour, $C_{i}$ must turn out to be independent on the infrared regularisation. This is not the case here, because the effective theory does not correctly reproduce the hard-collinear infrared divergence. However, Eq. (28) shows that all infrared divergent terms have the same structure as the original current $\Gamma$, so that they can simply be absorbed into a redefinition of the the functions $\xi_{P}, \xi_{\perp}$ and $\xi_{\|}$, irrespective of their origin. (The same is true for the quark self-energy contributions; therefore we do not need to calculate them explicitly.) In other words, the hard-collinear contributions preserve the HQET/large-recoil symmetries and can hence be disregarded in the discussion of symmetry-breaking corrections.

We therefore find it convenient to define the factorisation scheme (or renormalisation conventions for the "soft form factors") by imposing the condition that

$$
f_{+} \equiv \xi_{P}, \quad V \equiv \frac{M+m_{V}}{M} \xi_{\perp}, \quad A_{0} \equiv \frac{E}{m_{V}} \xi_{\|},
$$

hold exactly to all orders in perturbation theory. (Such a "physical" scheme is similar to defining the quark parton distribution to be the structure function $F_{2}$ in deep inelastic scattering to all orders in perturbation theory.) Having fixed the factorisation scheme, we insert, for a given current $\Gamma$, Eq. (28) into Eq. (11) and express the result in terms of the uncorrected (tree-level) soft form factors $\xi_{P}^{(0)}, \xi_{\perp}^{(0)}$ and $\xi_{\|}^{(0)}$. The relation between these uncorrected form factors and the corrected ones $\xi_{P}, \xi_{\perp}$ and $\xi_{\|}$is determined by Eq. (29) and by eliminating $\xi_{P}^{(0)}, \xi_{\perp}^{(0)}$ and $\xi_{\|}^{(0)}$, we finally express all other form factors in terms of $\xi_{P}, \xi_{\perp}$ and $\xi_{\|}$. The result reads

$$
\begin{aligned}
& f_{0}=\frac{2 E}{M} \xi_{P}\left(1+\frac{\alpha_{s} C_{F}}{4 \pi}[2-2 L]\right)+\frac{\alpha_{s} C_{F}}{4 \pi} \Delta f_{0}, \\
& f_{T}=\frac{M+m_{P}}{M} \xi_{P}\left(1+\frac{\alpha_{s} C_{F}}{4 \pi}\left[\ln \frac{m_{b}^{2}}{\mu^{2}}+2 L\right]\right)+\frac{\alpha_{s} C_{F}}{4 \pi} \Delta f_{T}
\end{aligned}
$$

for the remaining form factors of pseudoscalar mesons and

$$
\begin{aligned}
A_{1}= & \frac{2 E}{M+m_{V}} \xi_{\perp}+\frac{\alpha_{s} C_{F}}{4 \pi} \Delta A_{1}, \\
A_{2}= & \frac{M}{M-m_{V}}\left(\xi_{\perp}-\xi_{\|}\left(1+\frac{\alpha_{s} C_{F}}{4 \pi}[-2+2 L]\right)\right)+\frac{\alpha_{s} C_{F}}{4 \pi} \Delta A_{2}, \\
T_{1}= & \xi_{\perp}\left(1+\frac{\alpha_{s} C_{F}}{4 \pi}\left[\ln \frac{m_{b}^{2}}{\mu^{2}}-L\right]\right)+\frac{\alpha_{s} C_{F}}{4 \pi} \Delta T_{1}, \\
T_{2}= & \frac{2 E}{M} \xi_{\perp}\left(1+\frac{\alpha_{s} C_{F}}{4 \pi}\left[\ln \frac{m_{b}^{2}}{\mu^{2}}-L\right]\right)+\frac{\alpha_{s} C_{F}}{4 \pi} \Delta T_{2}, \\
T_{3}= & \xi_{\perp}\left(1+\frac{\alpha_{s} C_{F}}{4 \pi}\left[\ln \frac{m_{b}^{2}}{\mu^{2}}-L\right]\right) \\
& -\xi_{\|}\left(1+\frac{\alpha_{s} C_{F}}{4 \pi}\left[\ln \frac{m_{b}^{2}}{\mu^{2}}-2+4 L\right]\right)+\frac{\alpha_{s} C_{F}}{4 \pi} \Delta T_{3}
\end{aligned}
$$


for the remaining form factors of vector mesons. Here we introduced the abbreviation

$$
L=-\frac{2 E}{M-2 E} \ln \frac{2 E}{M}
$$

with $L \rightarrow 1$ for $E \rightarrow M / 2\left(q^{2} \rightarrow 0\right)$. In defining $L$ we identified the $b$ quark mass $m_{b}$ and the $B$ meson mass $M$, since the difference is a $1 / M$ effect.

The form factors receive a further additive correction from the interaction with the spectator quark, indicated by $\Delta F_{i}$ in Eqs. (30)-(37). This correction will be calculated in the next subsection, see Eqs. (56), (60), (61) below. The tensor form factors $f_{T}, T_{1,2,3}$ are scale-dependent, since the defining tensor currents are not conserved.

\subsection{Hard spectator interaction}

\subsubsection{General considerations}

As discussed above a further correction at order $\alpha_{s}$ arises from the spectator interaction shown in Figs. 1]c and d. To leading order in $1 / M$ we can restrict ourselves to the two-particle light-cone distribution amplitudes of the $B$ meson and the light meson.

The momenta of the $b$ quark and the spectator antiquark in the $\bar{B}$ meson are chosen as

$$
p_{b}^{\mu}=m_{b} v^{\mu}, \quad l^{\mu}=\frac{l_{+}}{2} n_{+}^{\mu}+l_{\perp}^{\mu}+\frac{l_{-}}{2} n_{-}^{\mu}
$$

respectively, and

$$
k_{1}^{\mu}=u E n_{-}^{\mu}+k_{\perp}^{\mu}+\frac{\vec{k}_{\perp}^{2}}{4 u E} n_{+}^{\mu}, \quad k_{2}^{\mu}=\bar{u} E n_{-}^{\mu}-k_{\perp}^{\mu}+\frac{\vec{k}_{\perp}^{2}}{4 \bar{u} E} n_{+}^{\mu}
$$

denote the momenta of the quark and antiquark in the light meson. As usual we have defined $\bar{u}=1-u$. Note that all components of the spectator momentum $l$ are of order $\Lambda_{\mathrm{QCD}}$, while $k_{1,2}$ are of order $M$ along the $n_{-}$-direction with transverse components of order $\Lambda_{\mathrm{QCD}}$.

The contribution of Figs. 11c and d to the heavy-to-light current matrix elements is now given by the convolution formula

$$
\langle L|\bar{q} \Gamma b| B\rangle^{(\mathrm{HSA})}=\frac{4 \pi \alpha_{s} C_{F}}{N_{C}} \int_{0}^{1} d u \int_{0}^{\infty} d l_{+} M_{\beta \gamma}^{B} M_{\delta \alpha}^{L} \mathcal{T}_{\alpha \beta \gamma \delta}^{\Gamma} .
$$

Here $\Gamma$ denotes an arbitrary Dirac matrix in the heavy-to-light current, and $\mathcal{T}_{\alpha \beta \gamma \delta}^{\Gamma}$ is the hard-scattering amplitude, to be calculated from the Feynman graphs in Figs. 11c and d. Dirac indices $\alpha, \beta, \gamma, \delta$ are written explicitly, while the colour trace has already been performed.

The relevant non-perturbative bound state dynamics of the initial and final mesons is encoded in the two-particle light-cone projectors $M^{B}$ and $M^{L}$ (not to be confused with the matrices $\mathcal{M}_{B}$ and $\mathcal{M}_{L}$ used in Eq. (12)). The expressions for these projectors are obtained after Fourier transformation to momentum space of the light-cone expansion of matrix elements of quark-antiquark operators. For light pseudoscalar and vector mesons the relevant details can be found in Ref. [22] and Ref. [23], respectively, and the complete 
expressions are summarised in Appendix A. For pseudoscalar mesons with momentum $p^{\prime}$ we have

$$
M_{\delta \alpha}^{P}=\frac{i f_{P}}{4} \not p^{\prime} \gamma_{5} \phi(u)+\ldots
$$

where $f_{P}$ is the pseudoscalar decay constant; for vector mesons

$$
M_{\delta \alpha}^{V}=-\frac{i}{4}\left\{f_{\perp} \phi^{*} \not p^{\prime} \phi_{\perp}(u)+f_{V} \not p^{\prime} \frac{m_{V}}{E}\left(v \cdot \varepsilon^{*}\right) \phi_{\|}(u)+\ldots\right\}_{\delta \alpha}
$$

with $f_{V}$ and $f_{\perp}$ denoting the longitudinal and transverse vector meson decay constants, defined through

$$
\left\langle V\left(p^{\prime}, \varepsilon^{*}\right)\left|\bar{q} \gamma_{\mu} q^{\prime}\right| 0\right\rangle=-i f_{V} m_{V} \varepsilon_{\mu}^{*}, \quad\left\langle V\left(p^{\prime}, \varepsilon^{*}\right)\left|\bar{q} \sigma_{\mu \nu} q^{\prime}\right| 0\right\rangle=f_{\perp}\left(p_{\mu}^{\prime} \varepsilon_{\nu}^{*}-p_{\nu}^{\prime} \varepsilon_{\mu}^{*}\right) .
$$

The ellipses in Eqs. (42), (43) denote twist-3 two-particle contributions as specified in Appendix A.

The light-cone projectors for heavy mesons have not yet been discussed in full generality in the literature. As shown in Appendix B the projector we need is

$$
M_{\beta \gamma}^{B}=-\left.\frac{i f_{B} M}{4}\left[\frac{1+\not b}{2}\left\{\phi_{+}^{B}\left(l_{+}\right) \not h_{+}+\phi_{-}^{B}\left(l_{+}\right)\left(\not l_{-}-l_{+} \gamma_{\perp}^{\nu} \frac{\partial}{\partial l_{\perp}^{\nu}}\right)\right\} \gamma_{5}\right]_{\beta \gamma}\right|_{l=\frac{l_{+}}{2} n_{+}} .
$$

The derivative acts on the amplitude, expressed in terms of the spectator quark momentum $l$, and subsequently $l$ is set equal to its plus-component. (In writing Eq. (45) we have assumed the relation (112), which is valid only when one sets to zero the threeparticle contributions to $\phi_{-}^{B}\left(l_{+}\right)$. However, keeping the more general form (109) would not alter our result, since the symmetry-breaking correction turns out to involve only the distribution amplitude $\phi_{+}^{B}\left(l_{+}\right)$.)

In Feynman gauge, the hard-scattering amplitude is given by the expression

$$
\begin{aligned}
\mathcal{T}_{\alpha \beta \gamma \delta}^{\Gamma} & =\left[\Gamma \frac{m_{b}(1+\phi)+\not-\not k_{2}}{\left(m_{b} v+l-k_{2}\right)^{2}-m_{b}^{2}} \gamma_{\mu}+\gamma_{\mu} \frac{\not k_{1}+\not k_{2}-\not}{\left(k_{1}+k_{2}-l\right)^{2}} \Gamma\right]_{\alpha \beta} \frac{1}{\left(l-k_{2}\right)^{2}}\left[\gamma^{\mu}\right]_{\gamma \delta} \\
& \simeq\left[\Gamma \frac{m_{b}(1+\not)-\bar{u} E \not l_{-}}{4 \bar{u}^{2} l_{+} m_{b} E^{2}} \gamma_{\mu}+\gamma_{\mu} \frac{E \not \phi_{-}-\not}{4 \bar{u} l_{+}^{2} E^{2}} \Gamma\right]_{\alpha \beta}\left[\gamma^{\mu}\right]_{\gamma \delta} .
\end{aligned}
$$

To arrive at the second line we approximated the hard scattering amplitude by its leading term in the heavy quark limit, neglecting terms of order $\Lambda_{\mathrm{QCD}} / M$. The numerator of the second term, $E \not \chi_{-}-\not$, is an exception to this. Here the subleading term $l$ has to be kept, since the leading term is annihilated by the leading-twist light-cone projection operators: $\gamma^{\mu} M^{P, V} \gamma_{\mu} \not \phi_{-}=0$. As a consequence both terms in the second line of Eq. (46) are of order $1 /\left(M \Lambda_{\mathrm{QCD}}\right)$ in the heavy quark limit.

The denominator of the first term vanishes quadratically with $\bar{u}$. Assuming, as usual, that the leading-twist light-cone distribution amplitudes of the light mesons vanish only linearly with $\bar{u}$, the term $m_{b}(1+\psi)$ in the numerator generates a contribution that diverges logarithmically for $\bar{u} \rightarrow 0$. Hence the amplitude is dominated by small gluon virtualities, i.e. soft physics. In order to justify our factorisation ansatz (27), we have to show 
that the soft (endpoint dominated) contributions do not break the heavy quark/large recoil symmetries and can be accounted for by a redefinition of $\xi_{P, \perp, \|}$. After this redefinition the hard scattering term $\Phi_{B} \otimes T_{i} \otimes \Phi_{P}$ in (27) is free of soft (endpoint) singularities and can be computed consistently in the hard scattering approach. To demonstrate this, we insert $1=\left(\not \not_{+} \not \not_{-}\right) / 4+\left(\not \not_{-} \not \not_{+}\right) / 4$ in front of $\Gamma$ in the trace

$$
\begin{aligned}
& \operatorname{tr}\left(M^{L} \Gamma(1+\psi) \gamma^{\mu} M^{B} \gamma_{\mu}\right)=2 \operatorname{tr}\left(\psi M^{L} \Gamma M_{B}\right) \\
& \quad=\frac{1}{2} \operatorname{tr}\left(\psi M^{L} \not \phi_{+} \not \phi_{-} \Gamma M_{B}\right)+\frac{1}{2} \operatorname{tr}\left(\psi M^{L} \not \not_{-} \not \phi_{+} \Gamma M_{B}\right) .
\end{aligned}
$$

The first term is already of the form of Eq. (11), and therefore respects the heavy quark/large recoil symmetry relations, while the second term with $\not \phi_{-}$next to $M_{L}$ vanishes. This shows that the endpoint singularities can be factorised into the soft form factors as necessary for the validity of the factorisation formula (27). Consequently, the symmetry-breaking corrections are due to large-momentum transfer interactions.

However, a further check needs to be performed. Some of the twist-3 distribution amplitudes that contribute to $M^{L}$ do not vanish as $\bar{u} \rightarrow 0$ and generate terms that diverge linearly at the endpoint. The linear divergence produces an additional factor $M / \Lambda_{\mathrm{QCD}}$ that compensates the $1 / M$ suppression of the twist-3 distribution amplitudes. Therefore, twist-3 distribution amplitudes contribute at leading order to the soft part of the form factors. In order to justify our factorisation ansatz, we have to show that these endpoint contributions can also be absorbed into a renormalisation of $\xi_{P, \perp, \|}$. Furthermore, the expression $E \not x_{-}$in the second term of (46) is not annihilated by the twist-3 part of the projector $M^{L}$, and this provides another source of leading power contributions. This contribution must also be shown to satisfy the symmetry relations. The first of these two contributions can be treated in a manner analogous to Eq. (47). The second term in the second line of (47), which does not have the required symmetry-preserving structure, does not vanish, when twist-3 terms are included in $M^{L}$, but it turns out to be of order $\bar{u}$ as $\bar{u} \rightarrow 0$, hence not leading to the power-counting breaking linear divergence. Indeed, for a pseudoscalar meson, we obtain

$$
M^{P} \not \not_{-} \not \phi_{+}=-\frac{i f_{P}}{4} \mu_{P} \gamma_{5}\left(\phi_{p}+\frac{\phi_{\sigma}^{\prime}}{6}\right) \not \not_{-} \not \phi_{+}+\ldots,
$$

where the ellipsis denote the contributions from distribution amplitudes which vanish as $\bar{u} \rightarrow 0$. The important point is that while $\phi_{p}$ and $\phi_{\sigma}^{\prime}$ do not vanish at the endpoint, the combination of both which appears in Eq. (48) does. Similarly, we obtain

$$
\begin{gathered}
M^{V} \not \not_{-} \not \phi_{+}=-\frac{i m_{V}}{4}\left[f_{V}\left(\AA^{*}-\left(v \cdot \varepsilon^{*}\right) \not \phi_{-}\right)\left(g_{\perp}^{(v)}+\frac{1}{2} g_{\perp}^{(a)^{\prime}}\right)\right. \\
\left.+f_{\perp} \frac{m_{V}}{E}\left(v \cdot \varepsilon^{*}\right)\left(h_{\|}^{(t)}+\frac{1}{2} h_{\|}^{(s)^{\prime}}\right)\right] \not \phi_{-} \not \phi_{+}+\ldots,
\end{gathered}
$$

which also vanishes at $\bar{u}=0$ (see Appendix A). This shows that all power-counting breaking linearly divergent contributions can be accounted for by a renormalisation of $\xi_{P, \perp, \|}$. The second of the two contributions discussed above is easily seen to preserve the heavy quark/large recoil symmetries, since

$$
\operatorname{tr}\left(M_{L} \gamma^{\mu} \not \chi_{-} \Gamma M_{B} \gamma_{\mu}\right)=\operatorname{tr}\left(\gamma_{\mu} M_{L} \gamma^{\mu} \not \not_{-} \not \phi_{+} \not \not_{-} \Gamma M_{B}\right)
$$


has already the structure required by Eq. (11).

Before concluding this general discussion, we note that only the distribution amplitude $\phi_{+}^{B}\left(l_{+}\right)$enters the symmetry breaking (but computable) corrections. This is seen immediately for the first term in Eq. (46) since only the term $-\bar{u} E \eta_{-}$is relevant for the symmetry breaking terms. The $\not_{-}$annihilates the corresponding term in $M^{B}$, and since there is no dependence on $l_{\perp}, \Phi_{-}\left(l_{+}\right)$does not contribute. In the case of the second term of Eq. (46) we need to examine the $l$ term. The term proportional to $\not x_{-}$in the $B$-meson projector (45) drops out since the factor $\not \phi_{-}$can be anticommuted in such a way that it annihilates with $M_{\delta \alpha}^{L}$. But the derivative term in Eq. (45) survives and leaves a transverse Dirac matrix $\gamma_{\perp}^{\nu}$ to the left of the matrix $\Gamma$. Inserting $1=\left(\not \not_{+} \not \eta_{-}\right) / 4+\left(\not \not_{-} \not_{+}\right) / 4$, the "wrong" projector $\left(\not \not_{-} \not \not_{+}\right) / 4$ is annihilated by the leading twist terms in $M^{L}$, since $\gamma_{\perp}^{\nu}$ anti-commutes with $\not_{ \pm}$. The remaining terms involving $\Phi_{-}\left(l_{+}\right)$then preserve the heavy quark/large recoil symmetries.

To summarise this subsection, the hard-scattering contributions, calculated from Eq. (41) fall into two classes: soft contributions that formally diverge at the endpoints $\bar{u} \rightarrow 0$ (or $l_{+} \rightarrow 0$ ) but obey the symmetry relations predicted by Eq. (11), and hard contributions that show regular behaviour at the endpoint and (potentially) break the symmetries. These general considerations will be verified by the explicit calculation that follows below.

\subsection{2 $\quad B \rightarrow P$ form factors}

The hard-scattering contributions to the current matrix elements for $B \rightarrow P$ transitions are calculated using Eqs. (41), (46) together with the light-cone projection operators. The form factors are then determined by comparing the result with the definitions in Eqs. (11) and (21).

To give an example, the result for the form factor $f_{+}$reads

$$
\begin{aligned}
& f_{+}^{(\mathrm{HSA})}=\frac{\alpha_{s} C_{F}}{4 \pi} \frac{\pi^{2} f_{B} f_{P} M}{N_{C} E^{2}} \int_{0}^{1} d u \int_{0}^{\infty} d l_{+}\left\{\frac{4 E-M}{M} \frac{\phi(u) \phi_{+}^{B}\left(l_{+}\right)}{\bar{u} l_{+}}\right. \\
& \left.+\frac{(1+\bar{u}) \phi(u) \phi_{-}^{B}\left(l_{+}\right)}{\bar{u}^{2} l_{+}}+\frac{\mu_{P}}{2 E}\left[\frac{\left(\phi_{p}(u)-\phi_{\sigma}^{\prime}(u) / 6\right) \phi_{+}^{B}\left(l_{+}\right)}{\bar{u}^{2} l_{+}}+\frac{4 E \phi_{p}(u) \phi_{+}^{B}\left(l_{+}\right)}{\bar{u} l_{+}^{2}}\right]\right\}
\end{aligned}
$$

Similar expressions are obtained for $f_{0}$ and $f_{T}$. The three terms in the second line of Eq. (52) have endpoint singularities for $\bar{u} \rightarrow 0$ and/or $l_{+} \rightarrow 0$. As discussed in the previous subsection these terms preserve the symmetry structure predicted by Eq. (11). In our factorisation scheme we can absorb these contributions into $\xi_{P}$ without having to specify a regularisation procedure for the endpoint singularities. In fact, due to the renormalisation convention Eq. (29) the entire correction displayed in Eq. (52) is absorbed into $\xi_{P}$. The important point is that terms with the structure of those in the second line (which cannot be computed with standard hard scattering methods) then do not appear in the other two form factors $f_{0}$ and $f_{T}$, whereas a term with the structure of that of the first line (which is computable) does, as will be seen below.

It is worth emphasizing again that all four terms in Eq. (52) are of the same order $\left(\Lambda_{\mathrm{QCD}} / M\right)^{3 / 2}$ with respect to the $1 / M$ power counting. In the current context this 
reflects the observation made in Ref. [3] that in the QCD sum rule calculation some of the twist-3 distribution amplitudes contribute at leading power to the soft part of the form factor. (We can make contact with the result of Ref. [3] by expanding the light-cone distribution amplitudes around $\bar{u}=0$. Identifying

$$
I_{2} \leftrightarrow \frac{\alpha_{s} C_{F}}{4 \pi} \frac{2 \pi^{2} f_{B}^{2} M}{N_{C}} \int \frac{d u}{\bar{u}} \int \frac{d l_{+}}{l_{+}} \phi_{-}^{B}, \quad I_{1} \leftrightarrow \frac{\alpha_{s} C_{F}}{4 \pi} \frac{2 \pi^{2} f_{B}^{2} M E}{N_{C}} \int \frac{d u}{\bar{u}^{2}} \int \frac{d l_{+}}{l_{+}} \phi_{+}^{B},
$$

and using the endpoint behaviour of the distribution amplitudes, we reproduce the result quoted in Eq. (87) of Ref. [3].) We also note that the soft contributions involve the $\phi_{-}^{B}$ amplitude of the $B$-meson.

The first term in Eq. (52) represents a genuine hard scattering correction which is dominated by gluon virtualities of order $\mu^{2} \simeq 2 E l_{+} \sim M \Lambda_{\mathrm{QCD}}$. At this order in $\alpha_{s}$, we need only two particular moments of the distribution amplitudes to compute this correction. For the light pseudoscalar meson, we need

$$
\left\langle\bar{u}^{-1}\right\rangle_{P}=\int d u \frac{\phi(u)}{\bar{u}}
$$

This is the same moment of the leading twist distribution amplitude that contributes to the $P \gamma$ transition form factor (see e.g. Ref. [24] and references therein). For the $B$ meson, we need

$$
\left\langle l_{+}^{-1}\right\rangle_{+}=\int d l_{+} \frac{\phi_{+}^{B}\left(l_{+}\right)}{l_{+}} .
$$

The same moment is also needed in $B \rightarrow \ell \nu \gamma$ decays [25], and it also determines the leading non-factorisable hard spectator corrections to $B \rightarrow \pi \pi$ decays [1, 2]. It is convenient to define the quantity

$$
\Delta F_{P}=\frac{8 \pi^{2} f_{B} f_{P}}{N_{C} M}\left\langle l_{+}^{-1}\right\rangle_{+}\left\langle\bar{u}^{-1}\right\rangle_{P}
$$

The theoretical uncertainties in the computation of the hard scattering correction due to the moments defined above and the $B$ meson decay constant are all contained in this quantity.

With the help of this notation, we now present the result for the hard scattering correction to $B \rightarrow P$ form factors, as defined by $\Delta f_{+, 0, T}$ in Eqs. (30), (31). The renormalisation convention (29) implies $\Delta f_{+} \equiv 0$ by definition. The other two quantities are then given by

$$
\Delta f_{0}=\frac{M-2 E}{2 E} \Delta F_{P}, \quad \Delta f_{T}=-\frac{M+m_{P}}{2 E} \Delta F_{P}
$$

Note that $\Delta f_{0}$ vanishes at $q^{2}=0(E=M / 2)$ as required on general grounds.

\subsection{3 $\quad B \rightarrow V$ form factors}

The analysis of the hard-scattering corrections to form factors for $B \rightarrow V$ transitions proceeds in the same way as for $B \rightarrow P$ decays. For instance, the contribution to the 
form factors $A_{0}$ and $T_{1}$ reads

$$
\begin{gathered}
A_{0}^{(\mathrm{HSA})}=\frac{\alpha_{s} C_{F}}{4 \pi} \frac{\pi^{2} f_{B} M}{N_{C} E^{2}} \int_{0}^{1} d u \int_{0}^{\infty} d l_{+}\left\{\frac{f_{V} \phi_{\|}(u) \phi_{+}^{B}\left(l_{+}\right)}{\bar{u} l_{+}}+\frac{(1+\bar{u}) f_{V} \phi_{\|}(u) \phi_{-}^{B}\left(l_{+}\right)}{\bar{u}^{2} l_{+}}\right. \\
\left.+\frac{m_{V} f_{\perp}}{2 E}\left[\frac{(-2 E) h_{\|}^{\prime}(s)(u) \phi_{+}^{B}\left(l_{+}\right)}{\bar{u} l_{+}^{2}}+\frac{\left(h_{\|}^{(t)}(u)-h_{\|}^{\prime}(s)(u) / 2\right) \phi_{+}^{B}\left(l_{+}\right)}{\bar{u}^{2} l_{+}}\right]\right\} \\
T_{1}^{(\mathrm{HSA})}=\frac{\alpha_{s} C_{F}}{4 \pi} \frac{\pi^{2} f_{B} M}{N_{C} E^{2}} \int_{0}^{1} d u \int_{0}^{\infty} d l_{+}\left\{\frac{2 E}{M} \frac{f_{\perp} \phi_{\perp}(u) \phi_{+}^{B}\left(l_{+}\right)}{\bar{u} l_{+}}+\frac{f_{\perp} \phi_{\perp}(u) \phi_{-}^{B}\left(l_{+}\right)}{\bar{u}^{2} l_{+}}\right. \\
\left.+\frac{m_{V} f_{V}}{2 E}\left[\frac{2 E\left(g_{\perp}^{(v)}(u)-g_{\perp}^{\prime}(a)(u) / 4\right) \phi_{+}^{B}\left(l_{+}\right)}{\bar{u} l_{+}^{2}}+\frac{\left(g_{\perp}^{(v)}(u)-g_{\perp}^{\prime}(a)(u) / 4\right) \phi_{+}^{B}\left(l_{+}\right)}{\bar{u}^{2} l_{+}}\right]\right\}
\end{gathered}
$$

The last three terms in $A_{0}$ and $T_{1}$ represent again a universal soft contribution that can be absorbed into $\xi_{\|}$and $\xi_{\perp}$, respectively. Only the first term gives rise to the symmetrybreaking correction. We introduce the quantities

$$
\begin{aligned}
\Delta F_{\|} & =\frac{8 \pi^{2} f_{B} f_{V}}{N_{C} M}\left\langle l_{+}^{-1}\right\rangle_{+}\left\langle\bar{u}^{-1}\right\rangle_{\|}, \\
\Delta F_{\perp} & =\frac{8 \pi^{2} f_{B} f_{\perp}}{N_{C} M}\left\langle l_{+}^{-1}\right\rangle_{+}\left\langle\bar{u}^{-1}\right\rangle_{\perp},
\end{aligned}
$$

where $\left\langle\bar{u}^{-1}\right\rangle_{\|}=\int d u \phi_{\|}(u) / \bar{u},\left\langle\bar{u}^{-1}\right\rangle_{\perp}=\int d u \phi_{\perp}(u) / \bar{u}$. The renormalisation convention (29) implies no correction to $A_{0}$ and $V$. The hard correction to the other $B \rightarrow V$ form factors, defined in Eqs. (32)-(37), reads

$$
\begin{aligned}
& \Delta A_{1}=0, \quad \Delta A_{2}=\frac{m_{V}}{E} \frac{M}{M-m_{V}} \frac{M(M-2 E)}{4 E^{2}} \Delta F_{\|} \\
& \Delta T_{1}=\frac{M}{4 E} \Delta F_{\perp}, \quad \Delta T_{2}=\frac{1}{2} \Delta F_{\perp}, \quad \Delta T_{3}=\frac{M}{4 E} \Delta F_{\perp}+\frac{m_{V}}{E}\left(\frac{M}{2 E}\right)^{2} \Delta F_{\|} .
\end{aligned}
$$

\subsection{Summary of corrections to form factor ratios}

The complete result for the form factors at order $\alpha_{s}$ is given by Eqs. (30)-(37), together with the expressions for the hard scattering correction in the previous subsection.

We summarise here how the form factor ratios (22)-(24) are modified by the symmetry-breaking corrections at order $\alpha_{s}$. For the $B \rightarrow$ pseudoscalar meson form factors we obtain

$$
\begin{aligned}
& \frac{f_{0}}{f_{+}}=\frac{2 E}{M}\left(1+\frac{\alpha_{s} C_{F}}{4 \pi}[2-2 L]+\frac{\alpha_{s} C_{F}}{4 \pi} \frac{M(M-2 E)}{(2 E)^{2}} \frac{\Delta F_{P}}{\xi_{P}}\right) \\
& \frac{f_{T}}{f_{+}}=\frac{m_{P}+M}{M}\left(1+\frac{\alpha_{s} C_{F}}{4 \pi}\left[\ln \frac{m_{b}^{2}}{\mu^{2}}+2 L\right]-\frac{\alpha_{s} C_{F}}{4 \pi} \frac{M}{2 E} \frac{\Delta F_{P}}{\xi_{P}}\right)
\end{aligned}
$$


The form factors for transitions between a $B$ meson and transversely or longitudinally polarized light vector mesons satisfy the relations

$$
\begin{gathered}
\frac{A_{1}}{V}=\frac{2 E M}{\left(M+m_{V}\right)^{2}}, \\
\frac{T_{1}}{V}=\frac{M}{2 E} \frac{T_{2}}{V}=\frac{M}{M+m_{V}}\left(1+\frac{\alpha_{s} C_{F}}{4 \pi}\left[\ln \frac{m_{b}^{2}}{\mu^{2}}-L\right]+\frac{\alpha_{s} C_{F}}{4 \pi} \frac{M}{4 E} \frac{\Delta F_{\perp}}{\xi_{\perp}}\right),
\end{gathered}
$$

and

$$
\begin{gathered}
\frac{\left(M+m_{V}\right) /(2 E) A_{1}-\left(M-m_{V}\right) / M A_{2}}{\left(m_{V} / E\right) A_{0}}= \\
1+\frac{\alpha_{s} C_{F}}{4 \pi}[-2+2 L]-\frac{\alpha_{s} C_{F}}{4 \pi} \frac{M(M-2 E)}{(2 E)^{2}} \frac{\Delta F_{\|}}{\left(E / m_{V}\right) \xi_{\|}}, \\
\frac{(M / 2 E) T_{2}-T_{3}}{\left(m_{V} / E\right) A_{0}}=1+\frac{\alpha_{s} C_{F}}{4 \pi}\left[\ln \frac{m_{b}^{2}}{\mu^{2}}-2+4 L\right]-\frac{\alpha_{s} C_{F}}{4 \pi}\left(\frac{M}{2 E}\right)^{2} \frac{\Delta F_{\|}}{\left(E / m_{V}\right) \xi_{\|}},
\end{gathered}
$$

respectively. The various quantities $\xi_{P, \perp, \|}, \Delta F_{P, \perp, \|}$ and $L$ are defined in Eqs. (29), (55),(59) and (38). The radiatively corrected form factor ratios (62)-(67) constitute the main result of this paper. Note that two relations, namely the ones between $A_{1}$ and $V$ and between $T_{1}$ and $T_{2}$ do not receive $\alpha_{s}$ corrections to leading order in the $1 / M$ expansion.

\section{Numerical analysis}

We now turn to the numerical analysis of the form factor ratios (62)-(67). We take a pion as a representative pseudoscalar meson and a $\rho$ meson as a representative vector meson. There is little theoretical uncertainty in the evaluation of the vertex correction. Assuming that the scale-dependent tensor form factors are renormalized at the scale $\mu_{1}=m_{b}$, the only uncertainty arises from the scale of $\alpha_{s}$, which we also take to be $\mu_{1}$.

The hard scattering correction is more difficult to estimate. Although it depends only on universal quantities, some of them, $\left\langle l_{+}^{-1}\right\rangle_{+}$in particular, are not well known. Note that the characteristic scale for the hard scattering correction is $\left(M \Lambda_{\mathrm{QCD}}\right)^{1 / 2}$ and all quantities in the hard scattering correction are evaluated at $\mu_{2}=1.47 \mathrm{GeV}$. We use the following input parameters:

Meson decay constants. The pion decay constant $f_{\pi}=131 \mathrm{MeV}$. For the $\rho$ meson decay constant we assume $f_{\rho}=198 \mathrm{MeV}$ and $f_{\rho, \perp}\left(\mu_{2}\right)=152 \mathrm{MeV}$, coincident with Ref. [26]. (We ignore a small difference due to the slightly different renormalisation scale.) Finally, $f_{B}=180 \mathrm{MeV}$. The uncertainty in the evaluation of the hard scattering correction coming from the decay constant is estimated to be around $\pm 15 \%$.

Light-cone distribution amplitudes. Since $\left\langle l_{+}^{-1}\right\rangle_{+} \sim 1 / \Lambda_{\mathrm{QCD}}$, but nothing else is known about this parameter at present, we estimate it to be $(0.2-0.5 \mathrm{GeV})^{-1}$ and take $(0.3 \mathrm{GeV})^{-1}$ as our central value. (Using the $B$ meson distribution amplitude suggested in Ref. [27], one obtains $\left\langle l_{+}^{-1}\right\rangle_{+}=(0.32 \mathrm{GeV})^{-1}$.) The situation is more favourable 
for the light mesons. The asymptotic distribution amplitude $\phi_{\pi}(u)=6 u \bar{u}$ is now favored by the CLEO data [28] on the $\pi \gamma$ form factor (see also Refs. 29, 24]), so that $\left\langle\bar{u}^{-1}\right\rangle_{\pi}=3$. For the $\rho$ meson we use the result for the second Gegenbauer moment quoted in Ref. [26], which leads to $\left\langle\bar{u}^{-1}\right\rangle_{\|}=3.48$ and $\left\langle\bar{u}^{-1}\right\rangle_{\perp}=3.51$. The uncertainty in the evaluation of the hard scattering correction coming from the meson distribution amplitudes is estimated to be around $\pm 50 \%$ with most of the uncertainty due to the $B$ meson.

Soft form factors. We also need an estimate for the absolute value of the functions $\xi_{\pi}, \xi_{\perp}$ and $\xi_{\|}$at large recoil in order to compute ratios such as $\Delta F_{P} / \xi_{P}$. Given the conditions (29), we use $f_{+}\left(q^{2}=0\right), V(0)$ and $A_{0}(0)$ as input and parametrise the energy dependence by the energy dependence of the soft form factor in the heavy quark/large recoil limit, Eq. (26), i.e. we take

$$
\left\{\xi_{P}, \frac{M+m_{V}}{M} \xi_{\perp}, \frac{E}{m_{V}} \xi_{\|}\right\}=\left\{f_{+}(0), V(0), A_{0}(0)\right\} \times\left(\frac{M}{2 E}\right)^{2}
$$

with

$$
\left\{f_{+}^{\pi}(0), V^{\rho}(0), A_{0}^{\rho}(0)\right\}=\{0.305,0.338,0.372\}
$$

from the QCD sum rule calculations of Refs. [26, 30]. The uncertainty in the evaluation of the hard scattering correction coming from the soft form factor is estimated to be around $\pm 20 \%$.

Combining these input parameters, we obtain

$$
\frac{\Delta F_{P}^{\pi}}{f_{+}^{\pi}(0)}=3.85, \quad \frac{\Delta F_{\perp}^{\rho}}{V^{\rho}(0)}=4.72, \quad \frac{\Delta F_{\|}^{\rho}}{A_{0}^{\rho}(0)}=5.54
$$

with an overall uncertainty of about $60 \%$. The result for the form factor ratios (62)-(67) is shown as the central solid curve in Fig. 2, using $\alpha_{s}\left(\mu_{1}\right)=0.22$ and $\alpha_{s}\left(\mu_{2}\right)=0.34$. The other two solid curves follow from multiplying the numbers in Eq. (70) by 0.4 and 1.6, respectively, and reflect the current theoretical uncertainty in evaluating the symmetry breaking correction. As a general rule, the hard scattering correction is larger than the vertex correction, and therefore the theoretical uncertainty remains significant. A determination of the $B$ meson parameter $\left\langle l_{+}^{-1}\right\rangle_{+}$would be very helpful to eliminate the single most important theoretical uncertainty. The typical size of the symmetry-breaking correction is of the order of $10 \%$ in the range $0 \leq q^{2} \leq M^{2} / 4$. An exception is the last relation (g), between $T_{2}, T_{3}$ and $A_{0}$, which might receive a negative correction of $30 \%$. Since the large recoil symmetries apply only for small $q^{2} \ll M^{2}$, the $q^{2}$ range shown is restricted to values smaller than $7 \mathrm{GeV}^{2}$.

It is instructive to compare our results with the QCD sum rule calculations. In Fig. 2 we plot the effective form factor parametrisation for $B \rightarrow \pi$ transitions from Ref. 30] and for $B \rightarrow \rho$ transitions from Ref. [26]. In general we find a fair agreement of the two results, as far as the magnitude and sign of the symmetry-breaking correction is concerned, with the exception of the ratios $T_{1} / V$ and $T_{2} / V$ (Fig. 2 $2 \mathrm{~d}$ and e). In particular, we note that in both approaches the relation between $A_{1}$ and $V$ (Fig. 2 c c) and the relation between $T_{1}$ and $T_{2}$ (Fig. 2 $\mathrm{d}$ vs. Fig. 2e) receive practically no corrections. The QCD sum rule calculations also include some $1 / M$ corrections, as well as quadratic meson mass effects, while the ratios (62)-(67) are strictly valid at leading order in the heavy quark 

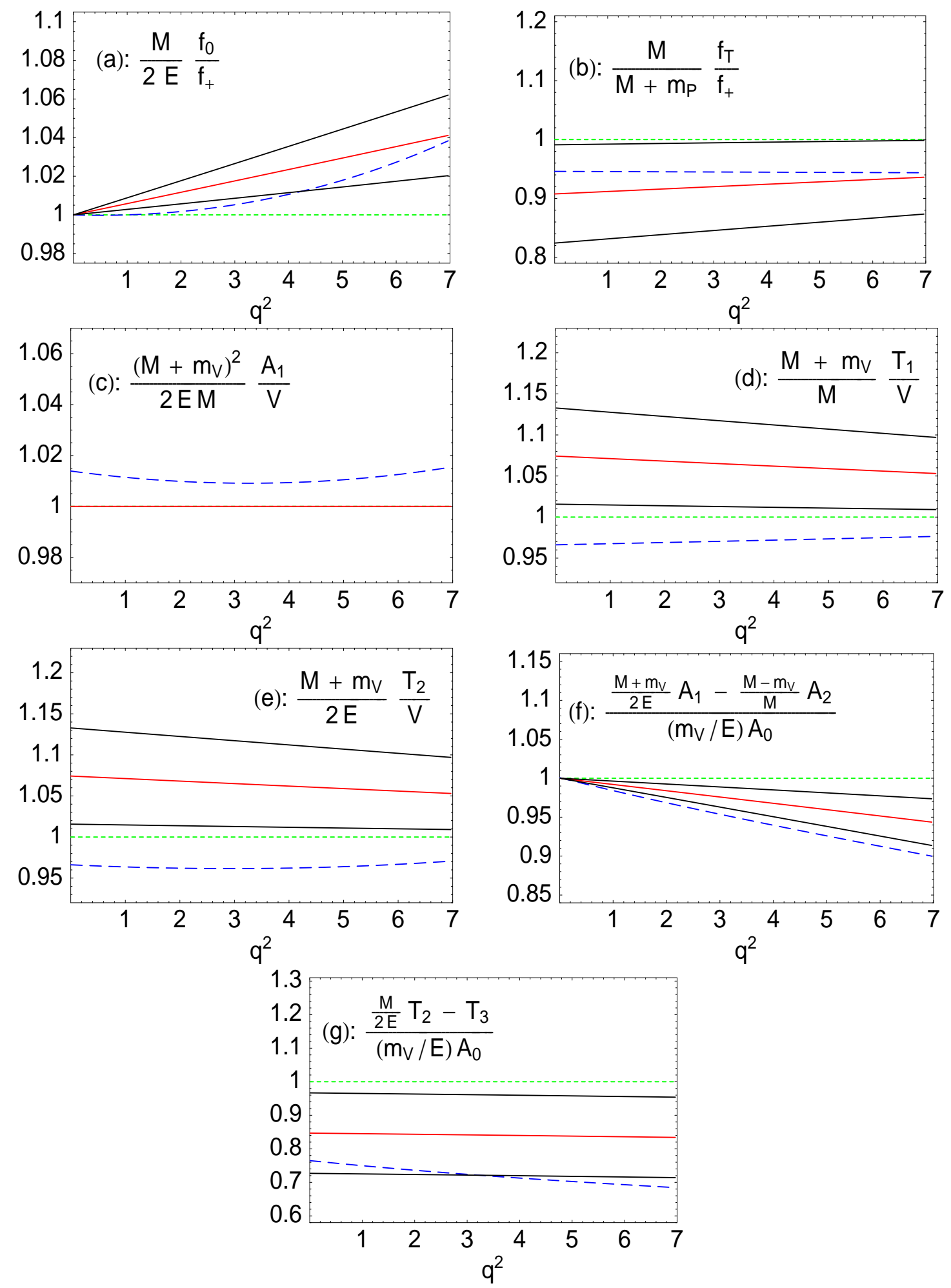

Figure 2: Corrections to symmetry relations as a function of $q^{2}$ (three solid lines, see text). Figs. (a)-(g) correspond to the form factor ratios in Eqs. (62)-(67) with overall scaling factors divided out, such that the symmetry limit (dotted lines) corresponds to a ratio equal to 1 independent on $q^{2}$. For illustration we show also results from QCD sum rules [30, 26] (dashed lines). Tensor form factors are renormalized at $\mu=m_{b}$. 
expansion. The two calculations are therefore not directly comparable at the level of $1 / M$ effects and this may explain the remaining numerical differences.

The advantage of the present approach over the QCD sum rule approach is that it does not require the duality assumption in the $B$ meson channel, but refers directly to the light-cone distributions of the $B$ meson. This is allows us to compute radiative corrections to the symmetry limit with less model-dependence than in the sum rule approach. The price for this is that the result depends on the parameter $\left\langle l_{+}^{-1}\right\rangle_{+}$, which may perhaps be constrained in the future, but remains poorly known for now. Furthermore, the duality assumption in the QCD sum rule method permits the calculation of the soft contribution to the form factors, which must be taken as an input, when one relies on symmetries and hard scattering methods only.

\section{Application to the forward-backward asymmetry in $B \rightarrow V \ell^{+} \ell^{-}$}

The forward-backward (FB) asymmetry in the decay $B \rightarrow V \ell^{+} \ell^{-}$(where $V$ is a vector meson, for example a $K^{*}$ meson) provides an interesting example, where the modelindependent form factor ratios derived above may be useful. Burdman [31] noted, using form factor models, that the location of the forward-backward asymmetry zero was nearly independent on the form factor models he considered. An explanation of this fact was given by Ali et al. [7], who noted that the form factor ratios on which the asymmetry zero depends are predicted free of hadronic uncertainties in the heavy quark/large energy limit considered in Ref. [3]. We are now in the position to discuss the effect of radiative corrections to the symmetry limit.

The decay $B \rightarrow K^{*} \ell^{+} \ell^{-}$is induced by the flavour-changing neutral current transition $b \rightarrow s \ell^{+} \ell^{-}$. In the Standard Model, after integrating out top quarks, $W$ and $Z$ bosons, it is described by the effective weak hamiltonian reviewed in detail in Ref. [6]. Let us begin the discussion by considering only the 'semi-hadronic' operators in the effective hamiltonian

$$
\mathcal{H}_{\mathrm{eff}}=-\frac{G_{F}}{\sqrt{2}} V_{t s}^{*} V_{t b} \sum_{i=7,9,10} C_{i}(\mu) \mathcal{O}_{i}(\mu)
$$

where

$$
\begin{gathered}
\mathcal{O}_{7}=\frac{e}{8 \pi^{2}} m_{b} \bar{s} \sigma^{\mu \nu}\left(1+\gamma_{5}\right) b F_{\mu \nu}, \\
\mathcal{O}_{9}=\left[\bar{s} \gamma^{\mu}\left(1-\gamma_{5}\right) b\right]\left[\bar{l} \gamma_{\mu} l\right], \quad \mathcal{O}_{10}=\left[\bar{s} \gamma^{\mu}\left(1-\gamma_{5}\right) b\right]\left[\bar{l} \gamma_{\mu} \gamma_{5} l\right],
\end{gathered}
$$

and $C_{i}(\mu)$ are the corresponding short-distance Wilson coefficients. The Wilson coefficients are calculable in the standard model, but they may also receive contributions from new particles in theories beyond the SM, and hence their experimental determination is of great interest. (Of course extensions of the standard model may introduce a larger set of operators as well.) The FB asymmetry zero in the decay $B \rightarrow K^{*} \ell^{+} \ell^{-}$provides a way to determine $C_{9}$. 
The forward-backward asymmetry is defined as

$$
\frac{d A_{\mathrm{FB}}}{d q^{2}}=\int_{0}^{1} d(\cos \theta) \frac{d^{2} \Gamma}{d q^{2} d \cos \theta}-\int_{-1}^{0} d(\cos \theta) \frac{d^{2} \Gamma}{d q^{2} d \cos \theta},
$$

where $\theta$ is the angle between the positively charged lepton and the $B$ meson in the $\ell^{+} \ell^{-}$ pair rest frame, and $q^{2}$ is the invariant mass of the lepton pair. The double-differential decay width is obtained by computing the matrix elements $\left\langle K^{*} \ell^{+} \ell^{-}\left|\mathcal{O}_{7,9,10}\right| \bar{B}\right\rangle$. The hadronic part of the matrix elements is parametrised in terms of the form factors in Eqs. (3)-(1). Without going into the details here (which can be found in Ref. [32, (7] or elsewhere), the FB asymmetry is found to be

$$
\begin{gathered}
\frac{d A_{\mathrm{FB}}}{d q^{2}} \propto C_{10}\left[C_{9} V\left(q^{2}\right) A_{1}\left(q^{2}\right)+\frac{m_{b}}{q^{2}} C_{7}\left(V\left(q^{2}\right) T_{2}\left(q^{2}\right)\left(M-m_{K^{*}}\right)\right.\right. \\
\left.\left.+A_{1}\left(q^{2}\right) T_{1}\left(q^{2}\right)\left(M+m_{K^{*}}\right)\right)\right]
\end{gathered}
$$

If this vanishes for a certain value $s_{0}=q_{0}^{2}$, then

$$
C_{9}=-\frac{m_{b}}{s_{0}} C_{7}\left\{\frac{T_{2}\left(s_{0}\right)}{A_{1}\left(s_{0}\right)}\left(M-m_{K^{*}}\right)+\frac{T_{1}\left(s_{0}\right)}{V\left(s_{0}\right)}\left(M+m_{K^{*}}\right)\right\}
$$

Making use of Eqs. (64), (65), we find that

$$
\frac{T_{2}\left(s_{0}\right)}{A_{1}\left(s_{0}\right)}\left(M-m_{K^{*}}\right)=\frac{T_{1}\left(s_{0}\right)}{V\left(s_{0}\right)}\left(M+m_{K^{*}}\right),
$$

including radiative corrections (neglecting, as always, terms of order $m_{K^{*}}^{2} / M^{2}$ ), so that Eq. (76) can be written as

$$
C_{9}=-\frac{2 M m_{b}}{s_{0}} C_{7}\left(1+\frac{\alpha_{s} C_{F}}{4 \pi}\left[\ln \frac{m_{b}^{2}}{\mu^{2}}-L\right]+\frac{\alpha_{s} C_{F}}{4 \pi} \frac{\Delta F_{\perp}}{\xi_{\perp}\left(s_{0}\right)}\right) .
$$

Consider first the leading order result. Even at leading order one cannot neglect the effect of four quark operators and the chromomagnetic dipole operator in the weak effective hamiltonian. Their effect is conventionally taken into account by defining "effective" Wilson coefficients, such that $C_{7} \rightarrow C_{7}^{\text {eff }}$ and $C_{9} \rightarrow \operatorname{Re}\left(C_{9}^{\text {eff }}\left(s_{0}\right)\right)$ in Eq. (78). $C_{9}^{\text {eff }}\left(s_{0}\right)$ is not a true short-distance quantity, acquiring $q^{2}$-dependence and an imaginary part, which turns Eq. (78) into an implicit equation for $s_{0}$. Assuming standard model values for the Wilson coefficients, the solution is $s_{0}=2.9 \mathrm{GeV}^{2}$ [7], which is small enough to justify the application of large recoil symmetries. The magnitude of the radiative correction to the symmetry limit of Eq. (78) can be deduced from Fig. 2 $\mathrm{d}$ do be $(6.5 \pm 5) \%$. This range can be considerably narrowed, when more information on the moment (54) of the $B$ meson distribution amplitude becomes available. Since $C_{7}^{\text {eff }}$ is already constrained to be close to its standard model value from the measurement of inclusive $b \rightarrow s \gamma$ decays, Eq. (78) provides an almost model-independent determination of $C_{9}$ as soon as $s_{0}$ is measured, and assuming that the matrix elements of the four quark operators can be computed to 


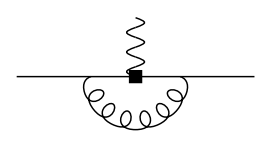

(a)

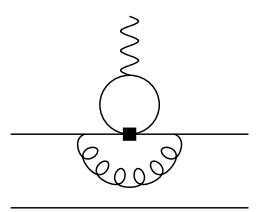

(c)

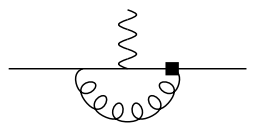

(e)

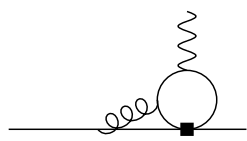

(g)

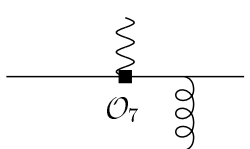

(b)

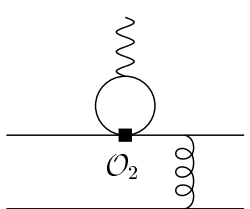

(d)

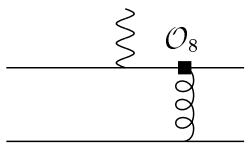

(f)

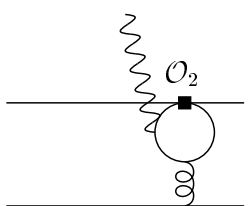

(h)

Figure 3: Various next-to-leading order contributions to the $B \rightarrow K^{*} \gamma^{*}$ matrix elements.

sufficient accuracy. (For completeness we note that the form factor ratios required for the present analysis are exactly those where there exists a discrepancy with the QCD sum rule result. From Fig. 2 $\mathrm{d}$, we also deduce that the QCD sum rule calculation of the relevant form factor ratios leads to a $3 \%$ reduction of $s_{0}$. This adds importance to clarifying the origin of this discrepancy.)

We should emphasize that Eq. (78) is not a complete result at order $\alpha_{s}$ even after replacing the Wilson coefficients $C_{7,9}$ by effective coefficients. A technical way to see this is to note that the $\mu$-dependent logarithm in Eq. (78), which arises from the scaledependent tensor form factor, does not compensate completely the renormalisation scale dependence of $C_{7}^{\text {eff }}$. There exist further corrections at order $\alpha_{s}$, originating from four quark operators and the chromomagnetic dipole operator in the weak effective hamiltonian, which cannot be expressed in terms of form factors, i.e. matrix elements of the type $\left\langle K^{*}|\bar{s} \Gamma b| \bar{B}\right\rangle$. Sample Feynman diagrams are shown in Fig. Be-g, compared to the diagrams in Fig. $3 \mathrm{a}-\mathrm{d}$, which do assume the structure of form factor matrix elements.

However, drawing upon the factorisation formula for non-leptonic $B$ decays [1, 2], we note that the matrix elements of all operators in the weak effective hamiltonian, including four quark operators, can be expressed as

$$
\left\langle K^{*} \ell^{+} \ell^{-}\left|\mathcal{O}_{i}\right| \bar{B}\right\rangle=C_{i} \xi+\Phi_{B} \otimes T_{i} \otimes \Phi_{K^{*}},
$$

i.e. in a form similar to Eq. (27). This allows us to compute the corrections of the 
type shown in Fig. 3e-g without introducing further non-perturbative parameters and to discuss exclusive radiative and semi-leptonic decays in a systematic way, comparable to the case of form factors and non-leptonic $B$ decays. This extension of the present work, and a complete discussion of the radiatively corrected FB asymmetry zero, will be presented elsewhere.

\section{Conclusion}

In this article we reconsidered the heavy quark/large recoil symmetries for heavy-tolight $B$ meson form factors (at large recoil) discussed first in Ref. [3]. We find that these symmetries, discussed originally for the soft parts of the form factors, survive radiative corrections in the sense that symmetry-breaking effects are dominated by hard scattering and therefore computable with standard methods. The structure of the corrections is tentatively summarised by the factorisation formula Eq. (27), which is similar to the factorisation formula for non-leptonic $B$ decays [1], 2]. However, much more theoretical work is needed to establish Eq. (27), or a variant thereof, beyond the calculation of symmetry-breaking effects.

The symmetries, and the calculation of symmetry-breaking effects, put the discussion of heavy-light form factors at large recoil on a similar conceptual footing as heavy-light form factors at small recoil or heavy-heavy form factors. In the symmetry limit (heavy quark limit) the three independent form factors for $B \rightarrow P$ transitions reduce to a single function $\xi_{P}$ for each pseudoscalar meson, and the seven independent form factors for $B \rightarrow V$ transitions reduce to two functions $\xi_{\perp}, \xi_{\|}$for each vector meson, corresponding to transverse or longitudinal polarization of the vector meson. Symmetry-breaking effects come from hard gluon corrections and fall into two classes: vertex corrections to the heavy-to-light current, which can be treated in an analogous way as in heavy quark effective theory, and hard rescattering with the spectator quark which is described by the hard-scattering approach and which involves light-cone distribution amplitudes of the participating mesons. The second class of corrections is a specifically new element of form factors at large recoil.

The numerical evaluation of the symmetry-breaking corrections typically yields $10 \%$ effects to form factor ratios; larger effects are possible for some form factor ratios, while two ratios do not receive any correction at order $\alpha_{s}$. There is at present a sizeable uncertainty in evaluating the hard-scattering correction, which seems to limit the usefulness of the present analysis. However, the major part of this uncertainty is due to a single moment of the $B$ meson distribution amplitude. This moment is a $B$-meson-universal quantity, and since only recently it has been realised that this quantity appears in many $B$ decays, we should expect this quantity to be determined much more accurately in the future.

The form factor relations at large recoil turn out to be strikingly useful for the forward-backward asymmetry in the exclusive decay $B \rightarrow K^{*} \ell^{+} \ell^{-}$, as already noted in Ref. [7]. Here we find symmetry-breaking corrections on the order of $5 \%$, but a definite conclusion must await the calculation of non-form factor type corrections at order $\alpha_{s}$. We plan to complete this task in a future publication. 


\section{Acknowledgements}

We would like to thank V.M. Braun, G. Buchalla and G.P. Korchemsky for helpful discussions.

\section{A Light-cone projections of light mesons}

\section{A.1 Twist-2 and -3 distribution amplitudes of pseudoscalars}

We follow the conventions of Ref. [22] in defining the light-cone distribution amplitudes of light pseudoscalar mesons:

$$
\begin{aligned}
\left\langle P\left(p^{\prime}\right)\left|\bar{q}(y) \gamma_{\mu} \gamma_{5} q(x)\right| 0\right\rangle & =-i f_{P} p_{\mu}^{\prime} \int_{0}^{1} d u e^{i\left(u p^{\prime} \cdot y+\bar{u} p^{\prime} \cdot x\right)} \phi(u) \\
\left\langle P\left(p^{\prime}\right)\left|\bar{q}(y) i \gamma_{5} q(x)\right| 0\right\rangle & =f_{P} \mu_{P} \int_{0}^{1} d u e^{i\left(u p^{\prime} \cdot y+\bar{u} p^{\prime} \cdot x\right)} \phi_{p}(u) \\
\left\langle P\left(p^{\prime}\right)\left|\bar{q}(y) \sigma_{\mu \nu} \gamma_{5} q(x)\right| 0\right\rangle & =i f_{P} \mu_{P}\left(p_{\mu}^{\prime} z_{\nu}-p_{\nu}^{\prime} z_{\mu}\right) \int_{0}^{1} d u e^{i\left(u p^{\prime} \cdot y+\bar{u} p^{\prime} \cdot x\right)} \frac{\phi_{\sigma}(u)}{6} .
\end{aligned}
$$

Here we defined $z=y-x$, and $f_{P}$ is the decay constant. The parameter $\mu_{P}=M_{P}^{2} /\left(m_{1}+\right.$ $m_{2}$ ) is proportional to the chiral condensate. (This definition holds for charged mesons, in which case the flavours of the light quarks with masses $m_{1}$ and $m_{2}$ are different.) $\phi(u)$ is the leading twist-2 distribution amplitude. All three distribution amplitudes are normalised to 1 , as can be seen by taking the limit $x \rightarrow y$. Above and in the following we implicitly assume that matrix elements are supplied with the appropriate path-ordered exponentials of gluon fields in order to make the definitions of distribution amplitudes gauge-invariant. The above definitions can be combined into

$$
\begin{aligned}
\left\langle P\left(p^{\prime}\right)\left|\bar{q}_{\alpha}(y) q_{\delta}(x)\right| 0\right\rangle=\frac{i f_{P}}{4} \int_{0}^{1} d u e^{i\left(u p^{\prime} \cdot y+\bar{u} p^{\prime} \cdot x\right)} \\
\quad \times\left\{\not p^{\prime} \gamma_{5} \phi(u)-\mu_{P} \gamma_{5}\left(\phi_{p}(u)-\sigma_{\mu \nu} p^{\prime \mu} z^{\nu} \frac{\phi_{\sigma}(u)}{6}\right)\right\}_{\delta \alpha}
\end{aligned}
$$

To obtain the momentum space projector $M_{\delta \alpha}^{L}$, which appears in Eq. (41), we take the Fourier transform of Eq. (81), using

$$
z^{\lambda} \rightarrow(-i) \frac{\partial}{\partial k_{1 \lambda}}=(-i)\left\{\frac{n_{+}^{\lambda}}{2 E} \frac{\partial}{\partial u}+\frac{n_{-}^{\lambda}}{2} \frac{\partial}{\partial k_{1}^{-}}+\frac{\partial}{\partial k_{\perp \lambda}}\right\}
$$

under Fourier transformation, where $k_{1}$ is the quark momentum defined in Eq. (40). The light-cone vectors $n_{ \pm}$are defined as after Eq. (10) and the transverse projection is defined with respect to these two vectors. The derivative is supposed to act on the hard scattering amplitude in the momentum space representation. The derivative with respect to the momentum fraction $u$ can be made to act on the light-cone distribution amplitude by partial integration; the second term in Eq. (82) drops out; the third term, 
which involves the derivative with respect to the transverse momentum, acts on the hardscattering amplitude before the collinear limit $k_{1}=u p^{\prime}=u E n_{-}$is taken. The light-cone projection operator of light pseudoscalar mesons in momentum space, including twist-3 two-particle contributions, then reads

$$
M_{\delta \alpha}^{P}=\frac{i f_{P}}{4}\left\{\not p^{\prime} \gamma_{5} \phi(u)-\mu_{P} \gamma_{5}\left(\phi_{p}(u)-i \sigma_{\mu \nu} n_{-}^{\mu} v^{\nu} \frac{\phi_{\sigma}^{\prime}(u)}{6}+i \sigma_{\mu \nu} p^{\prime \mu} \frac{\phi_{\sigma}(u)}{6} \frac{\partial}{\partial k_{\perp \nu}}\right)\right\}_{\delta \alpha} .
$$

A complete description of the pseudoscalar meson at the twist-3 level would also include three-particle quark-antiquark-gluon contributions, which we do not give here (see Ref. [22]).

The asymptotic limit of the leading twist distribution amplitude is $\phi(u)=6 u \bar{u}$. The twist-3 two-particle distribution amplitudes are completely determined by the threeparticle distributions via the equations of motions except for a single term. In the approximation that we set all three-particle distributions to zero (which is not an approximation that can be justified in any limit, but which is nonetheless useful to gain some insight in the structure of twist-3 two-particle contributions), the two twist-3 distribution amplitudes $\phi_{p}$ and $\phi_{\sigma}$ are related by the equations of motion

$$
\frac{u}{2}\left(\phi_{p}(u)+\frac{\phi_{\sigma}^{\prime}(u)}{6}\right)=\frac{\phi_{\sigma}(u)}{6}, \quad \frac{\bar{u}}{2}\left(\phi_{p}(u)-\frac{\phi_{\sigma}^{\prime}(u)}{6}\right)=\frac{\phi_{\sigma}(u)}{6},
$$

which imply $\phi_{p}(u)=1$ and $\phi_{\sigma}(u)=6 u \bar{u}$. Note that $\phi_{p}$ and $\phi_{\sigma}^{\prime}$ do not vanish at the endpoints.

\section{A.2 Twist-2 and -3 distribution amplitudes of vector mesons}

We follow Ref. [23] in defining the light-cone distribution amplitudes of light vector mesons with the exception that our convention for the outgoing light vector meson state differs by a factor $(-i)$ from the one of Ref. [23]. (This is necessary for consistency with the conventions used for the form factors in Eqs. (3)-(66).) We restrict ourselves again to the twist-2 and twist-3 two-particle amplitudes (neglecting terms suppressed by $m_{V}^{2} / E^{2}$ whenever they are not multiplied by the longitudinal polarisation vector), and define $z=y-x$ with $z^{2}=0$ as before. To correctly account for meson mass effects, we define the meson momentum to be $P^{\prime}$ with $P^{\prime 2}=m_{V}^{2}$ and the light-like vector $p_{\mu}^{\prime}=P_{\mu}^{\prime}-m_{V}^{2} z_{\mu} /\left(2 P^{\prime} \cdot z\right)$. The chiral-even amplitudes are given by

$$
\begin{gathered}
\left\langle V\left(P^{\prime}, \varepsilon^{*}\right)\left|\bar{q}(y) \gamma_{\mu} q(x)\right| 0\right\rangle=-i f_{V} m_{V} \int_{0}^{1} d u e^{i\left(u p^{\prime} \cdot y+\bar{u} p^{\prime} \cdot x\right)}\left\{p_{\mu}^{\prime} \frac{\varepsilon^{*} \cdot z}{p^{\prime} \cdot z} \phi_{\|}(u)+\varepsilon_{\perp \mu}^{*} g_{\perp}^{(v)}(u)\right\}, \\
\left\langle V\left(P^{\prime}, \varepsilon^{*}\right)\left|\bar{q}(y) \gamma_{\mu} \gamma_{5} q(x)\right| 0\right\rangle=i f_{V} m_{V} \epsilon_{\mu \nu \rho \sigma} \varepsilon^{* \nu} p^{\prime \rho} z^{\sigma} \int_{0}^{1} d u e^{i\left(u p^{\prime} \cdot y+\bar{u} p^{\prime} \cdot x\right)} \frac{g_{\perp}^{(a)}(u)}{4},
\end{gathered}
$$

with $f_{V}$ being the usual vector meson decay constant. The chiral-odd light-cone distribution amplitudes are given by

$$
\left\langle V\left(P^{\prime}, \varepsilon^{*}\right)\left|\bar{q}(y) \sigma_{\mu \nu} q(x)\right| 0\right\rangle=-f_{\perp} \int_{0}^{1} d u e^{i\left(u p^{\prime} \cdot y+\bar{u} p^{\prime} \cdot x\right)}\left\{\left(\varepsilon_{\perp \mu}^{*} p_{\nu}^{\prime}-\varepsilon_{\perp \nu}^{*} p_{\mu}^{\prime}\right) \phi_{\perp}(u)\right.
$$




$$
\begin{gathered}
\left.+\frac{m_{V}^{2} \varepsilon^{*} \cdot z}{\left(p^{\prime} \cdot z\right)^{2}}\left(p_{\mu}^{\prime} z_{\nu}-p_{\nu}^{\prime} z_{\mu}\right) h_{\|}^{(t)}(u)\right\} \\
\left\langle V\left(P^{\prime}, \varepsilon^{*}\right)|\bar{q}(y) q(x)| 0\right\rangle=-f_{\perp} m_{V}^{2} \varepsilon^{*} \cdot z \int_{0}^{1} d u e^{i\left(u p^{\prime} \cdot y+\bar{u} p^{\prime} \cdot x\right)} \frac{h_{\|}^{(s)}(u)}{2} .
\end{gathered}
$$

The chiral-odd amplitudes involve the (scale-dependent) transverse decay constant $f_{\perp}$. We have neglected terms proportional to the light-quark masses. The longitudinal and transverse projections of the polarization tensor are defined as [23]

$$
\varepsilon_{\| \mu}^{*} \equiv \frac{\varepsilon^{*} \cdot z}{P^{\prime} \cdot z}\left(P_{\mu}^{\prime}-\frac{m_{V}^{2}}{P^{\prime} \cdot z} z_{\mu}\right), \quad \varepsilon_{\perp \mu}^{*}=\varepsilon_{\mu}^{*}-\varepsilon_{\| \mu}^{*} .
$$

Note that the longitudinal projection of the polarization vector counts as $\mathcal{O}\left(E / m_{V}\right)$. Eqs. (85)-(88) can be combined into the expression

$$
\begin{aligned}
& \left\langle V\left(P^{\prime}, \lambda\right)\left|\bar{q}_{\alpha}(y) q_{\delta}(x)\right| 0\right\rangle=-\frac{i}{4} \int_{0}^{1} d u e^{i\left(u p^{\prime} \cdot y+\bar{u} p^{\prime} \cdot x\right)} \\
& \times\left\{f_{V} m_{V}\left(p_{\mu}^{\prime} \frac{\varepsilon^{*} \cdot z}{p^{\prime} \cdot z} \phi_{\|}(u)+\oiint_{\perp}^{*} g_{\perp}^{(v)}(u)+\epsilon_{\mu \nu \rho \sigma} \varepsilon^{* \mu} p^{\prime \rho} z^{\sigma} \gamma^{\mu} \gamma_{5} \frac{g_{\perp}^{(a)}(u)}{4}\right)\right. \\
& \left.\quad+f_{\perp}\left(\oiint_{\perp}^{*} \not p^{\prime} \phi_{\perp}(u)-i \frac{m_{V}^{2} \varepsilon \cdot z}{\left(p^{\prime} \cdot z\right)^{2}} \sigma_{\mu \nu} p^{\prime \mu} z^{\nu} h_{\|}^{(t)}(u)-i m_{V}^{2} \varepsilon^{*} \cdot z \frac{h_{\|}^{(s)}(u)}{2}\right)\right\}_{\delta \alpha} .
\end{aligned}
$$

To perform the Fourier transform we first express the previous equation in terms of the $z$-independent vectors $P^{\prime}$ and $\epsilon^{*}$. The Fourier transform of the terms with $p^{\prime} \cdot z=P^{\prime} \cdot z$ in the denominator can be treated by partial integration, leading to integrals over the distribution amplitudes. Potential surface terms vanish as a consequence of the relations (98) and (100) below (provided that additional gluon contributions vanish as well). After the Fourier transform is taken we introduce the two light-like vectors $n_{ \pm}$, and write $P_{\mu}^{\prime}=E n_{-\mu}+m_{V}^{2} n_{+\mu} /(4 E)$. The transverse plane is now defined with respect to the two vectors $n_{ \pm}$. We then obtain for the momentum space representation of the vector meson light-cone projection:

$$
M_{\delta \alpha}^{V}=M_{\delta \alpha \|}^{V}+M_{\delta \alpha \perp}^{V}
$$

with

$$
\begin{aligned}
M_{\|}^{V}= & -\frac{i f_{V}}{4} \frac{m_{V}\left(\varepsilon^{*} \cdot n_{+}\right)}{2 E} E \not \phi_{-} \phi_{\|}(u)-\frac{i f_{\perp} m_{V}}{4} \frac{m_{V}\left(\varepsilon^{*} \cdot n_{+}\right)}{2 E}\left\{-\frac{i}{2} \sigma_{\mu \nu} n_{-}^{\mu} n_{+}^{\nu} h_{\|}^{(t)}(u)\right. \\
& \left.-i E \int_{0}^{u} d v\left(\phi_{\perp}(v)-h_{\|}^{(t)}(v)\right) \sigma_{\mu \nu} n_{-}^{\mu} \frac{\partial}{\partial k_{\perp \nu}}+\frac{h_{\|}^{\prime}(s)(u)}{2}\right\}\left.\right|_{k=u p^{\prime}}
\end{aligned}
$$

and

$$
\begin{aligned}
M_{\perp}^{V}= & -\frac{i f_{\perp}}{4} E \phi_{\perp}^{*} \not \phi_{-} \phi_{\perp}(u)-\frac{i f_{V} m_{V}}{4}\left\{\oint_{\perp}^{*} g_{\perp}^{(v)}(u)\right. \\
& -E \int_{0}^{u} d v\left(\phi_{\|}(v)-g_{\perp}^{(v)}(v)\right) \not \phi_{-} \varepsilon_{\perp \mu}^{*} \frac{\partial}{\partial k_{\perp \mu}}
\end{aligned}
$$




$$
\left.+i \epsilon_{\mu \nu \rho \sigma} \varepsilon_{\perp}^{* \nu} n_{-}^{\rho} \gamma^{\mu} \gamma_{5}\left[n_{+}^{\sigma} \frac{g_{\perp}^{\prime(a)}(u)}{8}-E \frac{g_{\perp}^{(a)}(u)}{4} \frac{\partial}{\partial k_{\perp \sigma}}\right]\right\}\left.\right|_{k=u p^{\prime}}
$$

and where now

$$
\varepsilon_{\perp}^{\mu} \equiv \varepsilon^{\mu}-\frac{\varepsilon \cdot n_{+}}{2} n_{-}^{\mu}-\frac{\varepsilon \cdot n_{-}}{2} n_{+}^{\mu} .
$$

In the main body of the text we usually neglect power-suppressed higher-twist effects, i.e. we identify the meson momentum $P^{\prime}$ with $p^{\prime} \equiv E n_{-}$and set $\varepsilon^{*} \cdot n_{-}=0$.

The twist-3 distribution amplitudes are related to the twist-2 ones by WandzuraWilczek-type relations, namely [23]

$$
\begin{aligned}
& g_{\perp}^{(v)}(u)=\frac{1}{2}\left[\int_{0}^{u} \frac{\phi_{\|}(v)}{\bar{v}} d v+\int_{u}^{1} \frac{\phi_{\|}(v)}{v} d v\right]+\ldots \\
& g_{\perp}^{(a)}(u)=2\left[\bar{u} \int_{0}^{u} \frac{\phi_{\|}(v)}{\bar{v}} d v+u \int_{u}^{1} \frac{\phi_{\|}(v)}{v} d v\right]+\ldots
\end{aligned}
$$

for the chiral-even amplitudes, and

$$
\begin{aligned}
& h_{\|}^{(t)}(u)=(2 u-1)\left[\int_{0}^{u} \frac{\phi_{\perp}(v)}{\bar{v}} d v-\int_{u}^{1} \frac{\phi_{\perp}(v)}{v} d v\right]+\ldots, \\
& h_{\|}^{(s)}(u)=2\left[\bar{u} \int_{0}^{u} \frac{\phi_{\perp}(v)}{\bar{v}} d v+u \int_{u}^{1} \frac{\phi_{\perp}(v)}{v} d v\right]+\ldots
\end{aligned}
$$

for the chiral-odd amplitudes. The ellipses in Eqs. (95), (96) (and the following ones) indicate additional contributions from three-particle distribution amplitudes containing gluons and terms proportional to light quark masses, which we do not consider here. Eqs. (95), (96) also imply

$$
\begin{gathered}
\frac{g_{\perp}^{\prime(a)}(u)}{4}+g_{\perp}^{(v)}(u)=\int_{u}^{1} \frac{\phi_{\|}(v)}{v} d v+\ldots \\
\int_{0}^{u}\left(\phi_{\|}(v)-g_{\perp}^{(v)}(v)\right) d v=\frac{1}{2}\left[\bar{u} \int_{0}^{u} \frac{\phi_{\|}(v)}{\bar{v}} d v-u \int_{u}^{1} \frac{\phi_{\|}(v)}{v} d v\right]+\ldots,
\end{gathered}
$$

and

$$
\begin{gathered}
\frac{h_{\|}^{\prime}(s)(u)}{2}+h_{\|}^{(t)}(u)=-2 \bar{u}\left[\int_{0}^{u} \frac{\phi_{\perp}(v)}{\bar{v}} d v-\int_{u}^{1} \frac{\phi_{\perp}(v)}{v} d v\right]+\ldots \\
\int_{0}^{u}\left(\phi_{\perp}(v)-h_{\|}^{(t)}(v)\right) d v=u \bar{u}\left[\int_{0}^{u} \frac{\phi_{\perp}(v)}{\bar{v}} d v-\int_{u}^{1} \frac{\phi_{\perp}(v)}{v} d v\right]+\ldots
\end{gathered}
$$

Again all distribution amplitudes are normalized to unity. $\phi_{\perp}, \phi_{\|}, g_{\perp}^{(a)}$, and $h_{\|}^{(s)}$ vanish at the endpoints, whereas $g_{\perp}^{(v)}$, and $h_{\|}^{(t)}$ do not. 


\section{B Derivation of the $B$ meson projection}

\section{B.1 The momentum space projector}

In this appendix we derive the result for the $B$ meson projection operator stated in Eq. (45) starting from the two-particle light-cone matrix element in coordinate space. We follow the convention of Ref. [27] and introduce the two functions $\tilde{\phi}_{ \pm}^{B}(t)$ through the Lorentz decomposition of the following light-cone matrix element:

$$
\left\langle 0\left|\bar{q}_{\beta}(z) P(z, 0) b_{\alpha}(0)\right| \bar{B}(p)\right\rangle=-\frac{i f_{B} M}{4}\left[\frac{1+\psi}{2}\left\{2 \tilde{\phi}_{+}^{B}(t)+\frac{\tilde{\phi}_{-}^{B}(t)-\tilde{\phi}_{+}^{B}(t)}{t} \not\right\} \gamma_{5}\right]_{\alpha \beta} .
$$

We assume that $z^{2}=0$, defined $t=v \cdot z, p=M v$ and the path-ordered exponential

$$
P\left(z_{2}, z_{1}\right)=\mathrm{P} \exp \left(i g_{s} \int_{z_{2}}^{z_{1}} d z^{\mu} A_{\mu}(z)\right) .
$$

Eq. (101) is the most general parametrisation compatible with Lorentz-invariance and the heavy quark limit. The prefactor is chosen in such a way that for $z=0$ one obtains

$$
\left\langle 0\left|\bar{q}_{\beta}\left[\gamma^{\mu} \gamma_{5}\right]_{\beta \alpha} b_{\alpha}\right| \bar{B}(p)\right\rangle=i f_{B} M v^{\mu}
$$

if $\tilde{\phi}_{+}^{B}(t=0)=\tilde{\phi}_{-}^{B}(t=0)=1$.

Let us call $M(z)$ the matrix element in Eq. (101) and $A(z)(A(l))$ the hard scattering amplitude in coordinate (momentum) space. Then we obtain the momentum space projector $M^{B}$ of Eq. (45) through the identity

$$
\int d^{4} z M(z) A(z)=\left.\int \frac{d^{4} l}{(2 \pi)^{4}} A(l) \int d^{4} z e^{-i l z} M(z) \equiv \int_{0}^{\infty} d l_{+} M^{B} A(l)\right|_{l=\frac{l_{+}}{2} n_{+}},
$$

with $l$ decomposed as in Eq. (39),

$$
l^{\mu}=\frac{l_{+}}{2} n_{+}^{\mu}+\frac{l_{-}}{2} n_{-}^{\mu}+l_{\perp}^{\mu} .
$$

The factors $\not$ and $1 /(v \cdot z)$ that appear in Eq. (101) can be removed by having a derivative act on the hard scattering amplitude, and by partial integration, as in the case of the light meson distribution amplitudes. If we then introduce the momentum space distribution amplitudes through

$$
\tilde{\phi}_{ \pm}^{B}(t) \equiv \int_{0}^{\infty} d \omega e^{-i \omega t} \phi_{ \pm}^{B}(\omega)
$$

we obtain

$$
\begin{aligned}
& \int d^{4} z M(z) A(z)=-\frac{i f_{B} M}{4}\left[\frac { 1 + \psi } { 2 } \int _ { 0 } ^ { \infty } d \omega \left\{2 \phi_{+}^{B}(\omega)\right.\right. \\
&\left.\left.-\int_{0}^{\omega} d \eta\left(\phi_{-}^{B}(\eta)-\phi_{+}^{B}(\eta)\right) \gamma^{\mu} \frac{\partial}{\partial l_{\mu}}\right\} \gamma_{5}\right]\left._{\alpha \beta} A(l)_{\beta \alpha}\right|_{l=\omega v} .
\end{aligned}
$$


This is close to the desired expression except that $l=\omega v=\omega\left(n_{+}+n_{-}\right) / 2$. However, the hard scattering amplitude $A(l)$ for a decay into an energetic light meson moving in the $n_{-}$direction has the property that it is independent of $l_{-}$at leading order in the heavy quark expansion. More precisely, it can be written as

$$
A(l)=A^{(0)}\left(l_{+}\right)+l_{\perp}^{\mu} A_{\mu}^{(1)}\left(l_{+}\right)+O(1 / M) .
$$

Hence the $n_{-}$component of $v$ does not contribute and we may set $l=\omega n_{+} / 2$, which amounts to identifying $\omega$ and $l_{+}$in view of Eq. (39). Using

$$
\frac{\partial}{\partial l_{\mu}}=n_{-}^{\mu} \frac{\partial}{\partial l_{+}}+n_{+}^{\mu} \frac{\partial}{\partial l_{-}}+\frac{\partial}{\partial l_{\perp \mu}},
$$

(and dropping the derivative with respect to $l_{-}$), we obtain

$$
\begin{aligned}
M_{\beta \alpha}^{B}=-\frac{i f_{B} M}{4}[ & \frac{1+\psi}{2}\left\{\phi_{+}^{B}(\omega) \not \phi_{+}+\phi_{-}^{B}(\omega) \not x_{-}\right. \\
& \left.\left.\quad-\int_{0}^{l_{+}} d \eta\left(\phi_{-}^{B}(\eta)-\phi_{+}^{B}(\eta)\right) \gamma^{\mu} \frac{\partial}{\partial l_{\perp \mu}}\right\} \gamma_{5}\right]_{\alpha \beta} .
\end{aligned}
$$

It is understood that $l=l_{+} n_{+} / 2$ is set after performing the derivative. The $B$ meson light-cone projector assumes the form quoted in Eq. (45) after implementing the equation of motion constraint, Eq. (112), derived in the following subsection.

Notice that the independence of the hard scattering amplitude on $l_{-}$is exactly the property that guarantees that we need the bilocal matrix element (101) on the light-cone $\left(z^{2}=0\right)$.

\section{B.2 Equation of motion constraint}

We shall now show that the equation of motion for the light spectator quark relates $\phi_{-}^{B}\left(l_{+}\right)$to $\phi_{+}^{B}\left(l_{+}\right)$and three-particle quark-antiquark-gluon distribution amplitudes. This is similar to what happens for the twist-3 two-particle amplitudes of light vector mesons. In the approximation that the three-particle amplitudes are set to zero, we can determine $\phi_{-}^{B}\left(l_{+}\right)$in terms of $\phi_{+}^{B}\left(l_{+}\right)$. (These type of relations are sometimes referred to as "Wandzura-Wilczek relations" [33].)

In order to derive this relation we employ the equation of motion for the light quark in Eq. (101). Since the derivative with respect to $z_{\mu}$ has to be taken before the limit $z^{2} \rightarrow 0$ let us, for the moment, extend the definitions in Eq. (101) to the case $z^{2} \neq$

0 via $\tilde{\phi}_{ \pm}^{B}(t) \rightarrow \tilde{\phi}_{ \pm}^{B}\left(t, z^{2}\right)$. Requiring the right-hand side of Eq. (101) to vanish after application of $\left[\partial_{z_{2}}\right]_{\beta \gamma}$ (which is true only if the three-particle Fock-state $b \bar{q} g$ is neglected), and requiring $\tilde{\phi}_{ \pm}^{B}\left(t, z^{2}\right)$ to not vanish as $z^{2} \rightarrow 0$, we obtain

$$
\begin{aligned}
\frac{\partial \tilde{\phi}_{-}^{B}}{\partial t}+\left.\frac{1}{t}\left(\tilde{\phi}_{-}^{B}-\tilde{\phi}_{+}^{B}\right)\right|_{z^{2}=0} & =0 \\
\frac{\partial \tilde{\phi}_{+}^{B}}{\partial z^{2}}+\left.\frac{1}{4} \frac{\partial^{2} \tilde{\phi}_{-}^{B}}{\partial t^{2}}\right|_{z^{2}=0} & =0 .
\end{aligned}
$$


The second equation is uninteresting for our purpose. The first equation gives the desired relation between $\tilde{\phi}_{+}^{B}$ and $\tilde{\phi}_{-}^{B}$ in coordinate space. In terms of the momentum space distribution amplitudes, Eq. (110) reads

$$
\int_{0}^{l_{+}} d \eta\left(\phi_{-}^{B}(\eta)-\phi_{+}^{B}(\eta)\right)=l_{+} \phi_{-}^{B}\left(l_{+}\right) \quad \text { or } \quad \phi_{+}^{B}\left(l_{+}\right)=-l_{+} \phi_{-}^{B}\left(l_{+}\right),
$$

which is solved by

$$
\phi_{-}^{B}\left(l_{+}\right)=\int_{0}^{1} \frac{d \eta}{\eta} \phi_{+}^{B}\left(l_{+} / \eta\right) .
$$

In terms of Mellin moments one has $(N \geq 1)$

$$
\left\langle l_{+}^{N-1}\right\rangle_{+}=N,\left\langle l_{+}^{N-1}\right\rangle_{-}, \quad\left[\left\langle l_{+}^{N-1}\right\rangle_{ \pm} \equiv \int_{0}^{\infty} d l_{+} l_{+}^{N-1} \phi_{ \pm}^{B}\left(l_{+}\right)\right] .
$$

The relation (114) for $N=2$ has been derived independently in Ref. [27 from the equations of motions for the heavy quark and Lorentz invariance. This yields $\left\langle l_{+}\right\rangle_{+}=$ $2\left\langle l_{+}\right\rangle_{-}=4 / 3 \bar{\Lambda}$, where $\bar{\Lambda}$ is the leading contribution to the mass difference $M-m_{b}$ in HQET. For $N=3$ a similar analysis gives [27]

$$
\left\langle l_{+}^{2}\right\rangle_{+}=2 \bar{\Lambda}^{2}+\frac{2 \lambda_{E}^{2}+\lambda_{H}^{2}}{3}, \quad\left\langle l_{+}^{2}\right\rangle_{-}=\frac{2}{3} \bar{\Lambda}^{2}+\frac{\lambda_{H}^{2}}{3}
$$

where $\lambda_{E}$ and $\lambda_{H}$ parametrise the contributions of the chromoelectric and chromomagnetic fields to the mass difference $M-m_{b}$. Note that the relation (114) is again satisfied if we set $\lambda_{E}=\lambda_{H}$, and in particular if both quantities vanish which is equivalent to neglecting the three-particle Fock state as we have done. Grozin and Neubert [27] have also proposed the simple model distribution amplitudes $\phi_{+}^{B}\left(l_{+}\right)=l_{+} / l_{+0}^{2} \exp \left[-l_{+} / l_{+0}\right]$, $\phi_{-}^{B}\left(l_{+}\right)=1 / l_{+0} \exp \left[-l_{+} / l_{+0}\right]$ inspired by a QCD sum rule analysis. It is easy to see that they satisfy the relations (113), (114) exactly.

\section{References}

[1] M. Beneke et al., Phys. Rev. Lett. 83 (1999) 1914, hep-ph/9905312.

[2] M. Beneke et al., hep-ph/0006124.

[3] J. Charles et al., Phys. Rev. D60 (1999) 014001, hep-ph/9812358.

[4] N. Isgur and M.B. Wise, Phys. Lett. B232 (1989) 113.

[5] N. Isgur and M.B. Wise, Phys. Lett. B237 (1990) 527.

[6] G. Buchalla, A.J. Buras and M.E. Lautenbacher, Rev. Mod. Phys. 68 (1996) 1125, hep-ph/9512380.

[7] A. Ali et al., Phys. Rev. D61 (2000) 074024, hep-ph/9910221. 
[8] E. Eichten and B. Hill, Phys. Lett. B234 (1990) 511.

[9] B. Grinstein, Nucl. Phys. B339 (1990) 253.

[10] H. Georgi, Phys. Lett. B240 (1990) 447.

[11] M. Neubert, Phys. Rept. 245 (1994) 259, hep-ph/9306320.

[12] M.J. Dugan and B. Grinstein, Phys. Lett. B255 (1991) 583.

[13] G.P. Lepage and S.J. Brodsky, Phys. Rev. D22 (1980) 2157.

[14] A.V. Efremov and A.V. Radyushkin, Phys. Lett. B94 (1980) 245.

[15] V.L. Chernyak and I.R. Zhitnitsky, Nucl. Phys. B345 (1990) 137.

[16] A. Khodjamirian et al., Phys. Lett. B410 (1997) 275, hep-ph/9706303.

[17] E. Bagan, P. Ball and V.M. Braun, Phys. Lett. B417 (1998) 154, hep-ph/9709243.

[18] A. Szczepaniak, E.M. Henley and S.J. Brodsky, Phys. Lett. B243 (1990) 287.

[19] G. Burdman and J.F. Donoghue, Phys. Lett. B270 (1991) 55.

[20] R. Akhoury, G. Sterman and Y.P. Yao, Phys. Rev. D50 (1994) 358.

[21] M. Dahm, R. Jakob and P. Kroll, Z. Phys. C68 (1995) 595, hep-ph/9503418.

[22] V.M. Braun and I.E. Filyanov, Z. Phys. C48 (1990) 239.

[23] P. Ball et al., Nucl. Phys. B529 (1998) 323, hep-ph/9802299.

[24] T. Feldmann, Int. J. Mod. Phys. A15 (2000) 159, hep-ph/9907491.

[25] G.P. Korchemsky, D. Pirjol and T.M. Yan, Phys. Rev. D61 (2000) 114510, hepph/9911427.

[26] P. Ball and V.M. Braun, Phys. Rev. D58 (1998) 094016, hep-ph/9805422.

[27] A.G. Grozin and M. Neubert, Phys. Rev. D55 (1997) 272, hep-ph/9607366.

[28] CLEO Collaboration, J. Gronberg et al., Phys. Rev. D57 (1998) 33, hepex/9707031.

[29] P. Kroll and M. Raulfs, Phys. Lett. B387 (1996) 848, hep-ph/9605264.

[30] P. Ball, JHEP 09 (1998) 005, hep-ph/9802394.

[31] G. Burdman, Phys. Rev. D57 (1998) 4254, hep-ph/9710550.

[32] A. Ali, T. Mannel and T. Morozumi, Phys. Lett. B273 (1991) 505.

[33] S. Wandzura and F. Wilczek, Phys. Lett. B72 (1977) 195. 